\title{
BIRDS
}

\section{7th ANNUAL SASKATCHEWAN CHRISTMAS BIRD COUNT - 1998}

Compiled by Wayne C. Harris, Saskatchewan Environment and Resource Management, 350 Cheadle Street West, Swift Current, SK S9H 4G3

Counts were completed for 97 localities during the 1998 count period (December 18 - January 3). This is six less than last year but close to the long term average. Several long term counts were not done due to the windy conditions.

The weather leading into the Christmas count period was mild and generally snow free, at least in the south. Province wide snow cover did not occur until the end of December.

The mild temperatures ended on the first day of count period and then normal winter temperature prevailed. The average minimum temperature was $-24^{\circ} \mathrm{C}$ and the average high was $-18^{\circ}$, slightly below long term averages. Beauval recorded the coldest temperature $\left(-38^{\circ} \mathrm{C}\right)$ and Moose Jaw the warmest $\left(-7^{\circ} \mathrm{C}\right)$. Wind was probably the biggest factor for the counts.. Minimum daily wind speeds averaged almost $7 \mathrm{kmph}$ while the maximum average was more than 14 $\mathrm{kmph}$, both well above average. Several counts had sustained daily winds of $50 \mathrm{kmph}$. Table 1 summarizes weather conditions during the counts.

The total number of participants in the 1998 count was 735 , less than last years record number but still well above average. Observers walked more than $738 \mathrm{~km}$ in 376 hours, drove $12,842 \mathrm{~km}$ in 555 hours and spent 413 hours watching their feeders. Details of count effort and habitats covered are found in Table 2.

\section{The birds}

One hundred and eight species were recorded during the count period, 103 of these on count day with five more seen during the count period. In addition to these there were two recognizable subspecies (Table 3, 4 and 5). Although this is less than last years record 121 species it is till well above the average and exceeds the second highest total of 100 species recorded during both 1988 and 1996 counts.

Saskatoon topped the number of species recorded with 45 while Fort Walsh's was second with 40 . These were the only counts to reach 40 species and there were only six others that reached 30 species.

With the high number of species it is not surprising that the total number of individuals seen is also high. A total of 123,567 individuals were tallied second only to last years record.

The front runners in most abundant bird category were Snow Bunting and House Sparrow. The most frequently recorded species was Black-billed Magpie ( $98 \%$ of the counts) and Black-capped Chickadee (89\%). Surprisingly, Common Ravens were found on $71 \%$ of the counts far exceeding any other count year. 


\section{New Species}

One new species was recorded this year bringing the all time list to 168 . Two male Eastern Bluebirds seen during the count period feeding on chokecherries (accompanied by a female Mountain Bluebird) were Saskatchewan's first count record. These birds, found by Dave Phillips in the Craven count circle, were later observed by several other birders.

\section{Other rarities}

Table 4 lists the least frequent species on the counts and the rarest species are all in this table. A Pied-billed Grebe found at Fort Qu'appelle is the first record in a number of years. For the second year in a row Willow Ptarmigan have moved well south of their normal winter range with Love - Torch River having two. Two Killdeers at open springs in Saskatchewan Landing Provincial Park were only the third record with both other occurrences coming from Govenlock in 1982 and 1990. A Belted Kingfisher at Crooked Lake was also a third Christmas count record. The Mountain Chickadee at Cypress Hills Provincial Park was there for the third consecutive year. A female
Northern Cardinal at Fort Qu'appelle was only the fourth record and a Vesper Sparrow at Govenlock was a third record. A total of three Varied Thrushes were present this Christmas with singles at Waldheim count day and count period observations from Archerwill and Saskatoon.

\section{Population trends}

A number of new high counts were recorded (Table 6). The 24 Ruffed Grouse at Snowden was a new high. Saskatoon recorded new highs for both Black-billed Magpie and Black-capped Chickadee. The nine American Goldfinches at Kenosee Lake was exceptional not only for the high number but also for the general rarity of the species.

Although there were more Northern Goshawks this year compared to last year the long term trend is down and a similar pattern holds true for Prairie Falcon (Table 7). Snowy Owls populations were at there lowest number in the past decade but their long-term trend is stable. Winter finches were generally very low; most notable were Evening Grosbeaks, Pine Grosbeaks and both species of crossbills.

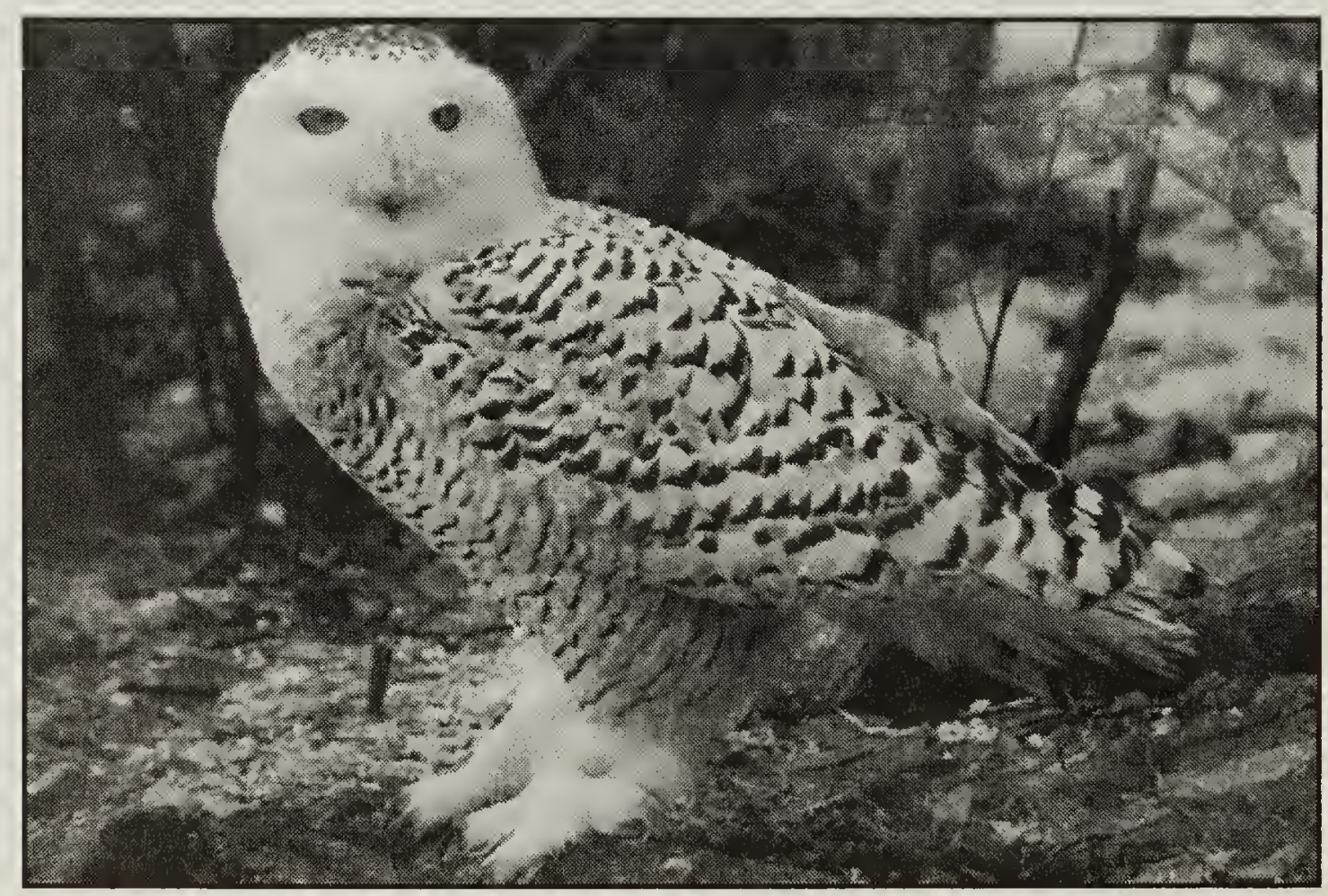


Both Gray Partridge and Ring-necked Pheasant have shown some recovery since the difficult winter of 1996-97 though numbers are still below the long term average. Ruffed Grouse numbers dropped slightly this year but are still above long term averages. Resident corvid populations are high most notably Black-billed Magpies and Common Ravens. Ravens were remarkably common south of the forest and their increasing winter distribution has been ongoing over the past two decades.
This year $71 \%$ of the counts recorded ravens with only those counts of the extreme southwest having no ravens.

House Finch numbers in Saskatchewan follow the continental pattern and continue to increase. First reported on a count in 1992, numbers and locations have increased dramatically in the past six years (Figure 1). Not only are they found on large urban centre counts but are now also on smaller town counts.

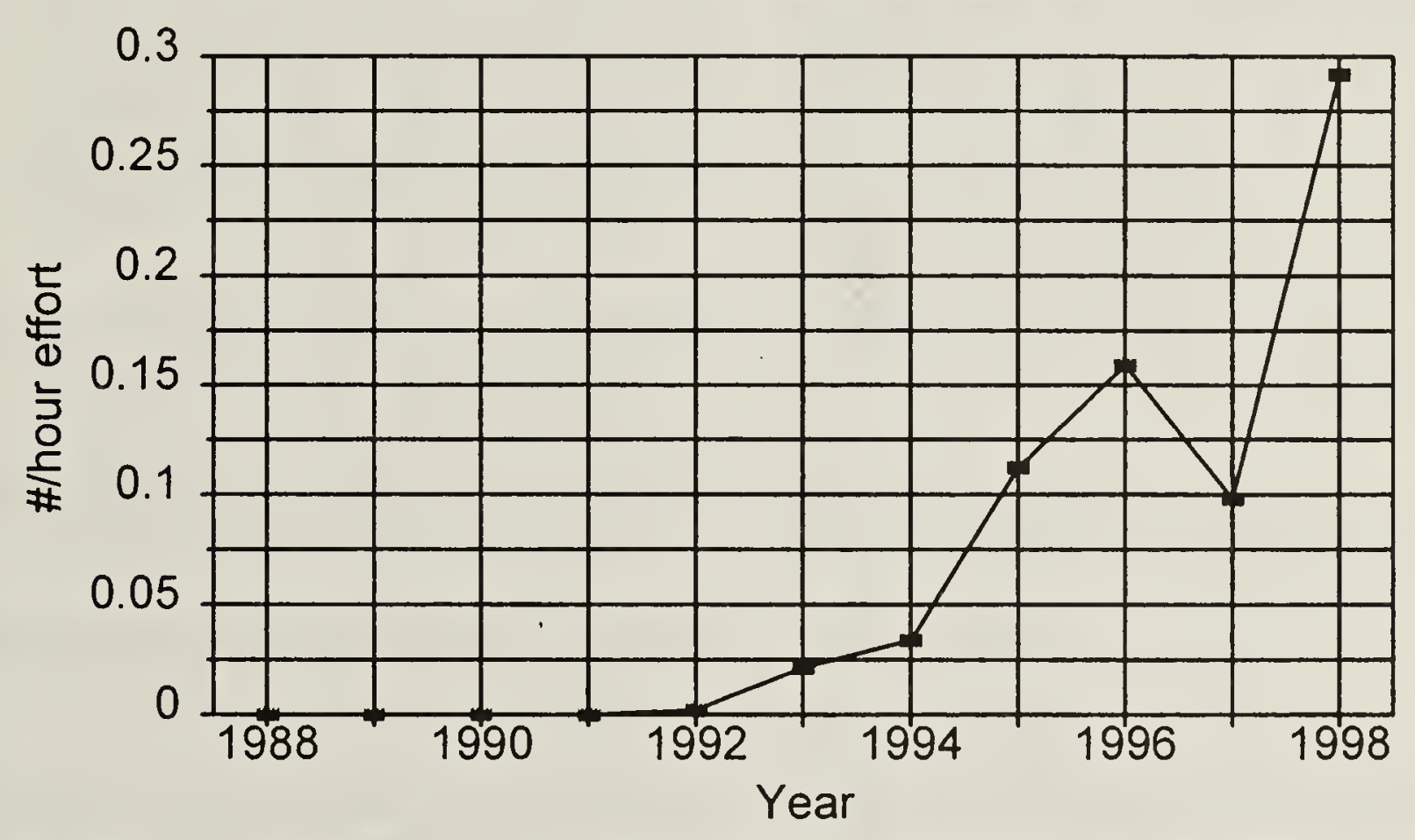

Figure 1. House Finch populations on Saskatchewan Christmas bird counts (1988-98)

\section{Count Areas and Participants}

(Names of compilers are in italics).

1. ARCHERWILL. Faye Black, Ken Folstad, Pauline Hnetka, Annette Kozak, Judy Revoy, Doris Slind.

2. ARMIT. Valeri Harris, Wayne Harris, Sheila Lamont.

3. BANGOR. Jean Hilton.

4. BEAUVAL. Jim Mills, Rick Sanderson.
5. BIGGAR. Dale Booth, Wayne Renaud, Guy Wapple, Robert Wapple, Sandra Wapple.

6. BIRCH HILLS. Marg Mareschal, Moe Mareschal, Don Weidl.

7. BRIGHTWATER RESERVOIR. Keith Hobson, Alan Smith.

8. BROADVIEW. Marlene Barnes, Ted Barnes, Doug Boivin, Barbara Weidl, Don Weidl, Lorraine Weidl, Tony Weidl. 
9. BROMHEAD. Martin Bailey, Carol Bjorklund.

10.CANDLE LAKE. Carmen Dodge, Marg Mareschal, Moe Mareschal, Don Weidl.

\section{CHITEK LAKE. Marcel Cornect.}

12.CLARK'S CROSSING. Carol Blenkin, Lucille Bradatsch, Erin Chatwell, Dave Cook, Louise Cook, Martha Guy, Tim Haughian, Darlene Hay, Jim Hay, Marlene Kalanack, Kay Krueger, Sarah Kuzmicz, Gerard Lahey, Doug McLean, Mack Miller, Garth Nelson, Menno Nickel, Hilda Noton, Lynn Oliphant, Randy Olson, Betty Penner, Stan Shadick, Rhonda Shewfelt, Martin Stoffel, Loren Teed, Ivor Thokle, Kirk Wallace, Michael Williams, Jim Wood.

13.COCHIN. Daryl Johannesen, Kaitlin Johannesen, Kristine Johannesen.

14.COLD LAKE, AB - PIERCELAND, SK. Jessica Roy, J. Frank Roy.

15.CORONACH. Valeri Harris, Wayne Harris, Sue McAdam.

16.CRAVEN. Margaret Belcher, Betty Binnie, Jim Cummings, Norman Herriot, Trevor Herriot, Phil Holloway, Bob Kreba, Kevin Moore, Ron Myers, Curtis Pollack, Dylan Pollack.

17.CROOKED LAKE. Bill Livsay, Mayta Livsay, Boyd Metzler, John Pollock, Dorothy Skene, Ed Skene.

18.CROOKED RIVER. Margaret Mehler, Morley Mehler.

19.CYPRESS HILLS PROVINCIAL PARK (Centre Block). Gary Churchill, Enid Cumming, John Douglas, Shirley Helmerson, Marilyn Scheller, Val Harris, Wayne Harris, Cameron
Lockerbie, Sue McAdam, Melody Nagel-Hisey, Steve Van Wilgenberg.

20.DUCK LAKE. Keith Hobson, Alan Smith.

21.DUVAL. George Herber, Lloyd Saul.

22.EASTEND. Robert Gebhardt, Joan Hodgins.

23. EMMA LAKE. Glen Hanson, Jean Hanson, Deanna Krug, Norman Krug.

24.ENDEAVOUR. Norman Harris.

25. ESTEVAN. Valeri Harris, Wayne Harris, Sue McAdam.

26. ESTUARY NORTH. Cathy Cocks, Dean Francis.

27.FENTON. Carman Dodge, Don Weidl.

28.FIFE LAKE. Martin Myers, Robert Rafuse.

29.FORT QU'APPELLE. James Armstrong, Phyllis Bordass, Errol Cochrane, Anne Davies, Doug Harman, Ronald Hooper, Kalvin Horseman, Alice Isfan, Vic Lamontagne, Kaye Lindgren, Jack Lowe, Allan Mlazgar, Webb Palmer, Paul Paquin, Lorne Rowell, Lloyd Talbot.

30.FORT WALSH. Pat Bulman, Valeri Harris, Wayne Harris, Ron Jensen, Burke Korol, Sheila Lamont, Susan McAdam, Wilkes Parsonage, Wayne Renaud, Guy Wapple.

31.GARDINER DAM. Pat Bulman, Terri Davidson, Greg Fenty, Valeri Harris, Wayne Harris, Mary Houston, Stuart Houston, Jeff Jensen, Ron Jensen, Burke Korol, Sheila Lamont, 
Cameron Lockerbie, Susan McAdam, Wayne Renaud, Guy Wapple.

32.GOOD SPIRIT LAKE. Bill Anaka, Joyce Anaka, Dorothy Riesz, Ray Riesz, Julia Wiwchar.

33.GOVENLOCK. Pat Bulman, Valeri Harris, Wayne Harris, Ron Jensen, Burke Korol, Sheila Lamont, Sue McAdam, Wayne Renaud, Guy Wapple, Robert Wapple.

34.GRASSLANDS NATIONAL PARK (NW). Alison Amero, Denis Blais, Shelly Duquette, Pat Fargey, Travis Godneir, Olaf Jensen, Dennis Madsen, Carol Masecar, Julie Parsonage.

35.GRAYSON. Charles Helm, Daniel Helm, Linda Helm, Karl Zimmer.

36. HARRIS. Wayne Renaud, Guy Wapple, Robert Wapple.

37. HEPBURN. Alice Pilatus, Phyllis Siemens.

38. HERSCHEL (Coal Mine Coulee). Wayne Renaud, Robert Wapple.

39. HOLBIEN. Chlorus Harris, Helen Harris.

40.HORSESHOE BEND (North of Kinistino). Daveen Berg, Dannelle Messer, Verna Messer, Alexis Simmons.

41.INDIAN HEAD. Carol Beaulieu, Denise Beaulieu, Graeme Beaulieu, Lauren Beaulieu, Mark Beaulieu, Vic Beaulieu, Irv Escott, David Gehl, Roberta Gehl, Gordon Howe, Dora Nichols, Ellen Varley, Ann Willerth, Gordon Willerth.
42.KAMSACK. George and Hazel Bernard, Agnes Betz, Mable Buceuk, Marlon Brock, Fred Chornoff, Lindee Dewores, Barb and Doug Elsaser, Bill Koroluik,Laura Loeppky, Leslie and Molly Pool, Kelsey and Ryley Rezansoff, Isabel Ritchie, Elsie Severson, Selma Smandych, Elenor Sookocheef, Joyce Ualow, I. Zeiben.

43. KELVINGTON. Pat Finnie, Dianne Sloan, Marguerite Sloan.

44.KENASTON. Lawrence Beckie.

45. KENOSEE LAKE. Boyd Metzler, John Pollock.

46.KINDERSLEY. Jean Harris, Keith Harris.

46. KINISTINO. Chris Derksen.

47. KINLOCH. Don Forbes, Doreen Forbes, Merle Jackson, Cliff Logan, Florence Olecsiewicz, Wilf Rodenberg.

48. KUTAWAGAN LAKE. Valeri Harris, Wayne Harris, Sheila Lamont.

49.LAST MOUNTAIN LAKE N.W.A. Anne Harris, Valeri Harris, Wayne Harris, Sheila Lamont.

50.LEADER (North). Daisy Meyers.

51.LEADER (South). Brenda Flood, John Flood.

52. LIVELONG. Sarah Pavka.

53.LOVE - TORCH RIVER. Ken Blaine, Lorna Blaine, Bert Dalziel, Duke Dalziel, Joan Dalziel, Kari Dalziel, Nora Dalziel, Sara Dalziel, Betty Donovan, Bruce Donovan, Eileen L'Heureux, Paul L'Heureux, George Lidster, Jean Lidster, Mildred Long, Lynn Matthews, William Matthews. 
54.LUSELAND. Kelly Cotter, Estelle Finley, Graeme Finley, Kerry Finley, Kim Finley, Laura Finley, Theron Finley, Val Finley, Bill Frey, Brent Honeker.

\section{MACDOWALL. Myron Barton.}

\section{MAYVIEW. Judith Graham.}

57. MEADOW LAKE. Bill Caldwell, Byron Golly, Stuart Golly, Tyler Golly, Lorraine Twidale, Joe Twidale, Bob Wilson.

\section{MELFORT. Frieda Markland.}

59.MELVILLE. Donna Alberts, Bill Barmby, Marion MacLean, Mildred Wotherspoon.

\section{MISSINIPE. Shirley Glass.}

61.MOOSE JAW. Elsie Adkins, Jack Adkins, Pam Albert, Ron and Vi Anderson, Vivien Brailean, Doug and Helen Brunsdon, Barry and Fern Dowse, Al Gurnsey, Kerry and Robert Handley, Bob and Pat Kern, Eve King, Cy and Leith Knight, Allan Lemieux, Bill McDonald, Connie and Hugh Mclntyre, Jean Anne and Len Mowchenko, Helen and Peter Norys, Lloyd Stewart, Arie Van Dorland.

62. MOOSE MOUNTAIN. Greg Bobbitt, Ross Douglas, Dick Gutfriend.

63.NAICAM. Lavawn Jensen.

64.NIPAWIN. Vi Budd, Joyce Christiansen, Bert Dalziel, Judith Friswell, Doug Pegg, Helen Pegg, Shirley Phillips, Jocelyn Thresher, Ruth Thresher.

\section{NISBET FOREST WEST (NW of} Crutwell). Kim Clark, Kiri Clark, Suzanne Clark, Ev Marshall.
66.PIKE LAKE. Nancy Allan, Tony Allen, Lawrence Beckie, Carol Blenkin, Patrick Bulman, Debbie Chatfield, David Cook, Louise Cook, Chantel Crossman, Mary Gilliland, Bob Girvan, Bernie Gollop, Mike Gollop, Robert Johanson, Regina Koenders, Kay Krueger, Gerard Lahey, Hilda Noton, Keith Pahl, Amanda Plante, Frank Roy, Maureen Scarfe, Doug Schneider, Marguerite Schneider, Stan Shadick, Martin Stoffel, Kirk Wallace, Ron Watson, Karen Wiebe, Michael Williams, Kay Willson, Phil Willson, Jim Wood, Lois Wooding, Adelle Young, Jackson Young, Nancy Young, John Yuen.

67.PORCUPINE PLAIN. Keith Larwood.

68.PREECEVILLE. Francis Buryk, Peter Buryk, Dallas Fairburn, Mary Mitchell, Muriel Mitchell, Russ Peet, Leona Pollock, Ella Smith, David Weiman.

69.PRINCE ALBERT. Les Anderson, Barry Bell, John Burt, Pamela Burt, Kim Clark, Carman Dodge, Keith Dodge, Philippe Gaudet, Joe Graumans, Alice Hambleton, Don Hambleton,Sandra Jewel, Eric Laing, Joyce Laing, Lillian Lubky, Jennie Mindiuk, Gerald Murphy, Helen Placsko, Les Senner, Winona Senner, Ted Snow, Beryl Wait, Don Wait.

70.PRINCE ALBERT NATIONAL PARK. Susan Carr, Allison Fisher, Bill Fisher, Kim Fisher, Ruby Fisher, Dan Frandsen, Jay Frandsen, Shanna Frith, Doug Gullickson, Scott Keesey, Brad Lloyd, Kurt Mazur, Bradley Muir, Adam Pidwerbeski, Lawrence Pidwerbeski, Samuel Pidwerbeski, Jeni Rudisill, Bob Turnbull, Dave Wieder, Cam Zimmer, Robin Zimmer. 
71. QU'APPELLE VALLEY DAM. Cameron Lockerbie, Frank Roy, Michael Williams, Phil Willson.

72.RAYMORE. Anne Harris, Valeri Harris, Wayne Harris, Sheila Lamont.

73.REGINA. Peter Ashcroft, Delmar Bamford, Margaret Belcher, Lionel Bonneville, Stephane Bonneville, Rick Douslin, Jim Elliott, Janet Galger, Jonathon Herriot, Kate Herriot, Trevor Herriot, Dale Hjertaas, Estelle Hjertaas, Paule Hjertaas, Phyllis Ilsley, Bob Kreba, Linda Langenbacher, Rheal Laroche, Catherine Ann Marr, Anne-Marie Murphy, Ron Meyers, Jean Ottosen, Katrina Ottosen, Jean Parker, Wayne Pepper, Brian Rainey, Curt Schroeder, Gerald Small, Kim Stevenson, Frank Switzer.

74.ROCKGLEN - BORDERLAND. Bob Rafuse.

75.ROUND LAKE (Qu'Appelle Valley). Pat Connolley, Doug Francis, Boyd Metzler, John Pollock, Pat Ward.

76.SASKATCHEWAN LANDING PROVINCIAL PARK. Val Harris, Wayne Harris, Jeff Jensen, Ron Jensen, Cameron Lockerbie, Susan McAdam.

77.SASKATCHEWAN RIVER FORKS. Carman Dodge, Don Weidl.

78.SASKATOON. Bruce Acton, Don Adams, Darlene Aikman, Nancy Allan, Tony Allen, Juhachi Asai, Kengo Asai, Rika Asai, Sumiko Asai, Bob Besant, Jeff Besant, Joyce Besant, Bruce Brotzel, Don Beazely, Mark Bidwell, Gladys Baxter, Howard Baxter, David Cook, Louise Cook, Eileen Dalglish, Ed Driver, Marg Driver, Randi Edmonds, Fran Eldridge, Melanie Elliott, Jeanine
Fleury, Margaret Freund, Martin Gerard, Marschall Gilliland, Mary Gilliland, Bernie Gollop, Maddie Gollop, Mike Gollop, Jean Gordon, Bob Green, Kaija Harris, Tim Haughian, Marty Helgerson, John Horton, David Houston, Donald Houston, Mary Houston, Stuart Houston, Marlene Kalanack, Gordon Koshinsky, Margaret Koshinsky, Barbara Kozmyk, Gerard Lahey, Catherine Laratte, Anna Leighton, Ted Leighton, Louise Roy Mark, Edna McCawley, Terry McCawley, Shirley McKercher, David Miller, Kathryn Miller, Meewasin Valley Authority staff (2), Bob Nelson, Garth Nelson, Lillian Nelson, Bruce Noton, Hilda Noton, Lynn Oliver, Keith Pahl, Amanda Plante, Garry Rector, Wayne Renaud, Edith Riekman, Allan Rosenberg, Marella Rosta, Andy Salisburg, Craig Salisburg, Lorienne Salisburg, Rob Salisburg, Maureen Scarfe, Doug Schmeiser, Susan Skinner, Jim Smart, Al Smith, Janice Solem, Angela Stern, Martin Stoffel, Flo Turcot, Hilda Voth, Heather Wagg, Kirk Wallace, Jim Wedgwood, George West, Colette Wheler, Michael Williams, Ben Willson, Jennifer Willson, Kay Willson, Phil Willson, Jim Wood, Judy Wood, Lois Wooding, Dan Zazelenchuk, Norman Zlotkin.

\section{SHAMROCK. Hugh Henry.}

80.SKULL CREEK. Catherine Bennetto, Eileen Bennetto, Ray Bennetto, Sharon Bennetto, Tim Bennetto, Doris Bircham, Ralph Bircham, Greeba Drever, Betty Mann, Robert Mann, Joanne Peterson, Larry Petterson, Harvey Wasilow.

81. SNOWDEN. Bonny Bailey, Rita Birhenthal, Elenor and Mike Bodnaruk, Irene Hagel, Karen Hagel, Jenny Johnson, Ralph Johnson, 
Violet Lien, Lorne Lindberg, Sharon Lindberg, Jack Pickett, Vera Schemenauer, Orville Shwetz, Vicky Shwetz, Bev Smears, Harold and Irene Thompson.

82.SPALDING. Bill Spizawka, Christina Spizawka, Jamie Spizawka, Kerry Spizawka, Velma Spizawka.

83.SPRUCE HOME. Sharon and Tom Dice.

84.SQUAW RAPIDS. Valeri Harris, Wayne Harris, Sheila Lamont, Guy Wapple.

85.SWIFT CURRENT. Evelyn Anderson, James Beattie, Noris Currie, Larry Dudragne, Hugh Henry, Jeff Jensen, Julie Jensen, Ron Jensen, Doreen Kreuger, Walt Kreuger, Arlene Lawson, Doug Lawson, Sue McAdam, Albert Otway, Ted Philipchuk, John Pound, Floyd Stinson, Irene Stinson, Kae Waters, John Weston, Pearl Weston, Verdella Zacharias.

86. TISDALE. George Curry, Marjorie Curry, Jenny Fretshaw, Vince Fretshaw, Joyce Mohr, Jim Wright, Mary Wright.

87.TOGO. Barb Elsasser, Doug Elsasser.

88. TURTLE LAKE. E.M. Robinson, Marie Robinson.

89.WALDHEIM. Barb Epp, Vicki Gossen.

90.WATSON. Doris Breker, Doreen MacDonald, Irene Sampson, Patricia Sterzuk, Doreen Weber.

91.WELDON. Ada Hawrish, Bernard Hawrish.
92.WELDON. Carmen Hanson.

93.WEYBURN. Leo Belanger, Sophie Belanger, Bob Cameron, Jeff Cameron, Lucille Cameron, Fred Garner, Dick Gutfriend, Kelly Kozij,Grace Kurtz, Cy Marcotte, Keith Sakatch, Norbert Schultz, Stewart Stairmand, Sid Trepoff.

94. WHITE BEAR. Darryl Jordheim, Floyd Jordheim, Sig Jordheim, Greg McCullock, Keely McCullock, Lynette McCullock.

95.WHITEWOOD. Ellen Blyth, Stephen Ede, Tom Ede, Joyce Jordan, Wilfred Jordens, Bernice Juzyniec, Felix Juzyniec, Jean Meadows, Boyd Metzler, John Pollock, Alfred Pritchard, Florence Pritchard, Lenore Santo, Diane Veresh, Pat Ward, Jean Wilson, Edwin Wyatt, Elain Wyatt.

96.YORKTON. Bill Anaka, Joyce Anaka, Elinor Hjertaas, Mark Kornder, Boyd Metzlar, Ray Riesz, Geoff Rushowick, Patrick Rushowick, Dorothy Skene, Ed Skene, Harold Wilkinson.

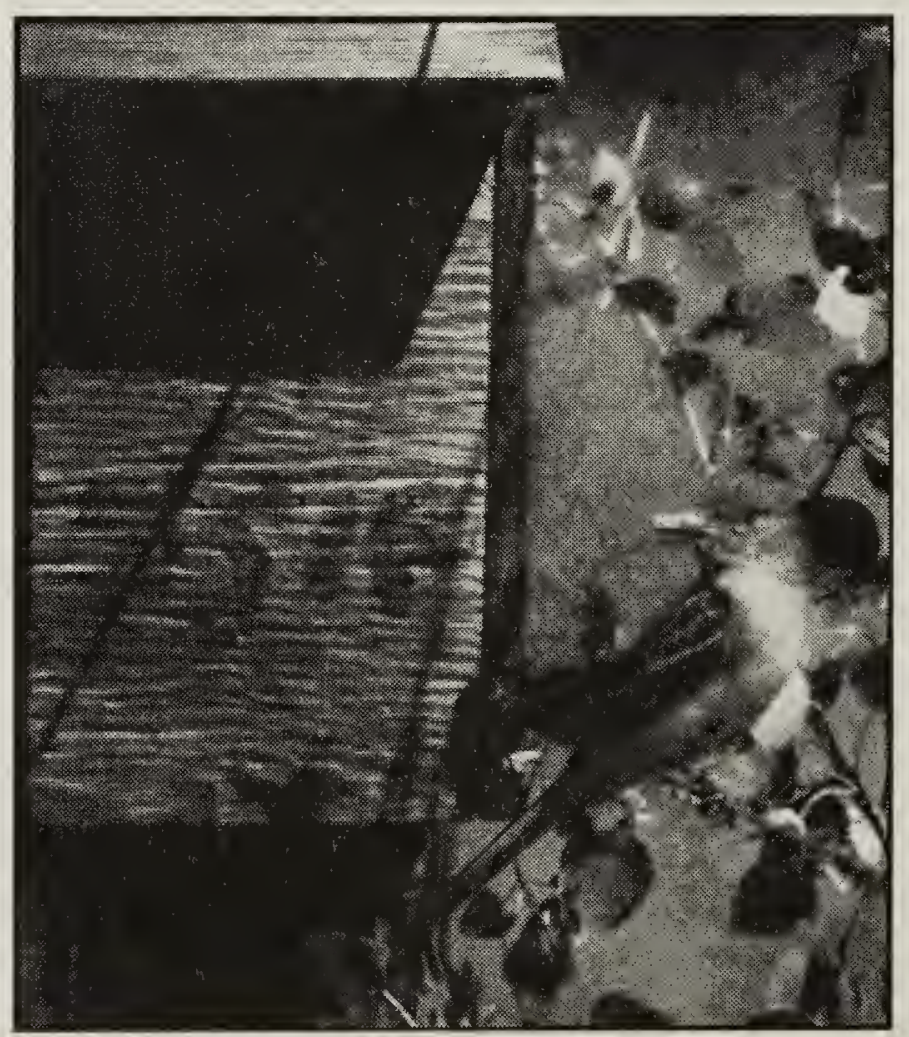

Great Crested Flycatcher

Ray Pettinger 


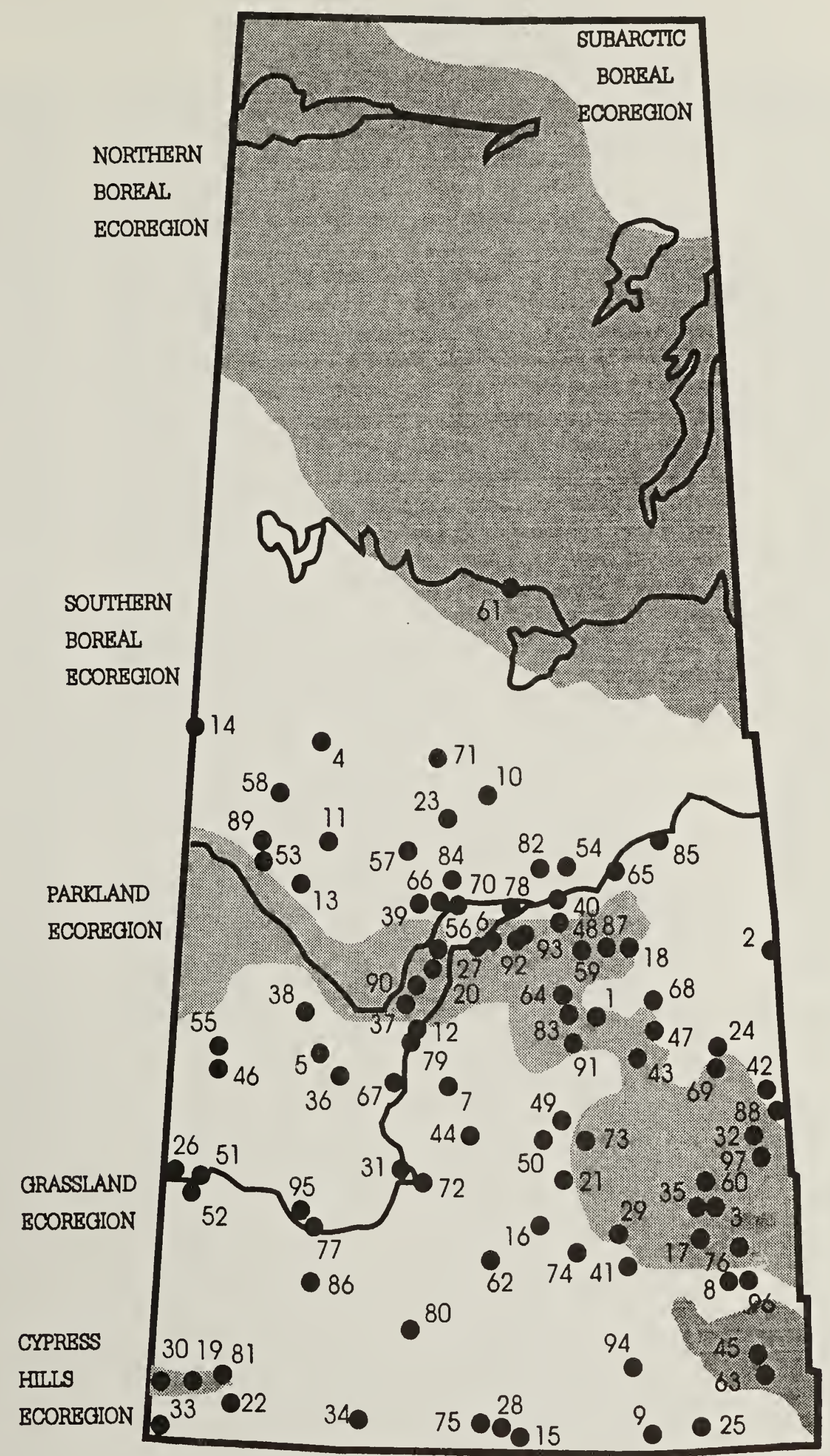

Figure 1.

Location of 1998 counts (numbers correspond to locality names in Tables) 


\begin{tabular}{|c|c|c|c|c|c|c|c|c|c|}
\hline COUNT & DATE & 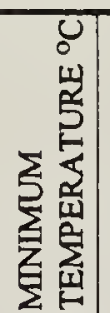 & 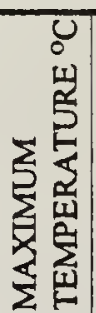 & 总突 & 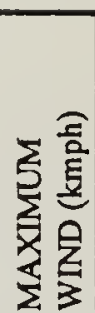 & 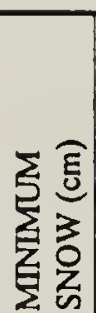 & 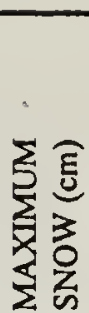 & SKY AM & SKY PM \\
\hline 1 ARCHERWILL & Dec. 29,1998 & -25 & -22 & 0 & 5 & 8 & 10 & clear & clear \\
\hline \begin{tabular}{l|l}
2 & ARMIT
\end{tabular} & Jan. 02,1999 & -30 & -25 & 2 & 15 & 5 & 10 & mostly clear & clear \\
\hline \begin{tabular}{l|l}
3 & BANGOR
\end{tabular} & Dec. 31,1998 & -32 & -25 & 20 & 25 & 10 & 15 & mostly clear & mostly clear \\
\hline 4 BEAUVAL & Jan. 03, 1999 & -38 & -23 & 0 & 4 & 38 & 45 & mostly clear & overcast \\
\hline 5 BIGGAR & Dec. 27,1998 & -15 & -12 & 0 & 16 & $\overline{0}$ & 8 & partly cloudy & partly cloudy \\
\hline 6 BIRCH HILLS & Dec. 22,1998 & -22 & -21 & 30 & 50 & 5 & 8 & mostly clear & partly cloudy \\
\hline 7 BRIGHTWATER & Dec. 21,1998 & -25 & -20 & 5 & 10 & 0 & 10 & mostly clear & mostly clear \\
\hline 8 BROADVIEW & Dec. 26,1998 & -21 & -15 & 5 & 10 & 3 & 8 & partly cloudy & partly cloudy \\
\hline 9 BROMHEAD & Dec. 31,1998 & -25 & -20 & 0 & 5 & 5 & 30 & mostly clear & partly cloudy \\
\hline 10 CANDLE LAKE & Dec. 30,1998 & -22 & -21 & 10 & 15 & 10 & 15 & overcast & partly cloudy \\
\hline 11 CHITEK LAKE & Dec. 20,1998 & -28 & -22 & 0 & 2 & 10 & 18 & mostly clear & mostly clear \\
\hline 12 CLARK'S & Dec. 19,1998 & -20 & -18 & 0 & 15 & 1 & 15 & overcast & mostly clear \\
\hline 13 COCHIN & Dec. 27, 1998 & -17 & -15 & 2 & 5 & 5 & 10 & partly cloudy & partly cloudy \\
\hline 14 COLD & Dec. 26,1998 & -17 & -15 & 0 & 10 & 10 & 15 & mostly clear & mostly clear \\
\hline 15 CORONACH & Dec. 23,1998 & -20 & -16 & 15 & 35 & 0 & 2 & clear & clear \\
\hline \begin{tabular}{l|l|}
16 & CRAVEN \\
\end{tabular} & Dec. 19,1998 & -24 & -20 & 10 & 15 & 20 & 45 & clear & mostly clear \\
\hline 17 CROOKED LAKE & Dec. 21,1998 & -30 & -25 & 30 & 40 & 10 & 13 & clear & clear \\
\hline 18 CROOKED & Dec. 28,1998 & -25 & -25 & 0 & 0 & 15 & 18 & mostly clear & mostly clear \\
\hline 19 CYPRESS HILLS & Dec. 30,1998 & -16 & -11 & 15 & 20 & 15 & 30 & partly cloudy & mostly clear \\
\hline 20 DUCK LAKE & Dec. 29,1998 & -30 & -20 & 0 & 5 & 0 & 20 & mostly clear & partly cloudy \\
\hline 21 DUVAL & Dec. 28,1998 & -29 & -22 & 0 & 9 & 0 & 15 & clear & mostly clear \\
\hline \begin{tabular}{l|l}
22 & EASTEND
\end{tabular} & Dec. 29,1998 & -15 & 0 & 0 & 0 & 0 & 18 & clear & clear \\
\hline 23 EMMA LAKE & Jan. 02, 1999 & -27 & -17 & 0 & 10 & 12 & 15 & clear & partly cloudy \\
\hline \begin{tabular}{l|l}
24 & ENDEAVOUR \\
\end{tabular} & Dec. 22,1998 & -22 & -19 & 15 & 20 & 15 & 25 & heavy fog & light fog \\
\hline 25 ESTEVAN & Dec. 24,1998 & -20 & -15 & 10 & 30 & 0 & 2 & clear & clear \\
\hline 26 ESTUARY & Jan. 03, 1999 & -23 & -17 & 5 & 10 & 5 & 10 & mostly clear & clear \\
\hline \begin{tabular}{l|l}
27 & FENTON
\end{tabular} & Dec. 21,1998 & -30 & -22 & 20 & 30 & 8 & 10 & mostly clear & mostly clear \\
\hline 28 FIFE LAKE & Dec. 29,1998 & -26 & -20 & 2 & 15 & 0 & 10 & mostly clear & mostly clear \\
\hline 29 FORT & Dec. 18,1998 & -18 & -10 & 20 & 40 & 3 & 5 & mostly clear & mostly clear \\
\hline 30 FORT WALSH & Dec. 19,1998 & -25 & -20 & 16 & 40 & 0 & 25 & partly cloudy & partly cloudy \\
\hline 31 GARDINER DAM & Dec. 21,1998 & -31 & -25 & 16 & 30 & 0 & 7 & clear & partly cloudy \\
\hline 32 GOOD SPIRIT & Jan. 03, 1998 & -34 & -24 & 5 & 15 & 8 & 25 & clear & clear \\
\hline 33 GOVENLOCK & Dec. 20,1998 & -26 & -15 & 5 & 25 & 0 & 0 & clear & mostly clear \\
\hline 34 GRASSLANDS & Dec. 21,1998 & -25 & -20 & 5 & 10 & 2 & 4 & clear & \\
\hline 35 GRAYSON & Dec. 26,1998 & -21 & -8 & 2 & 10 & 0 & 25 & partly cloudy & partly cloudy \\
\hline 36 HARRIS & Dec. 23, 1998 & -21 & -18 & 0 & 10 & 4 & 6 & mostly clear & partly cloudy \\
\hline 37 HEPBURN & Dec. 30,1998 & -21 & -21 & 0 & 0 & 8 & 12 & clear & clear \\
\hline \begin{tabular}{l|l}
38 & HERSCHAL
\end{tabular} & Dec. 29,1998 & -25 & -20 & 0 & 20 & 0 & 10 & partly cloudy & mostly clear \\
\hline 39 HOLBEIN & Dec. 27,1998 & -22 & 0 & 0 & 0 & 10 & 15 & light fog & light fog \\
\hline \begin{tabular}{l|l}
40 & HORSESHOE
\end{tabular} & Jan. 01, 1998 & -30 & -21 & 0 & 0 & 2 & 10 & mostly clear & clear \\
\hline 41 INDIAN HEAD & Dec. 27, 1998 & -20 & -15 & 10 & 15 & 25 & 30 & mostly clear & mostly clear \\
\hline 42 KAMSACK & Dec. 26,1998 & -30 & -28 & 40 & 50 & 30 & 60 & clear & clear \\
\hline \begin{tabular}{|l|l|}
43 & KELVINGTON \\
\end{tabular} & Dec. 31,1998 & -21 & -21 & 0 & 0 & 10 & 15 & & overcast \\
\hline \begin{tabular}{l|l}
44 & KENASTON \\
\end{tabular} & Dec. 21,1998 & -24 & -15 & 5 & 10 & 0 & 0 & mostly clear & partly cloudy \\
\hline 45 KENOSEE LAKE & Dec. 20,1998 & -26 & -22 & 15 & 20 & 10 & 25 & & clear \\
\hline \begin{tabular}{l|l}
46 & KINDERSLEY \\
\end{tabular} & Dec. 26,1998 & -22 & -18 & & & 0 & 1 & partly cloudy & overcast \\
\hline 47 KINISTINO & Dec. 26,1998 & -27 & -25 & 0 & 0 & 8 & 60 & clear & clear \\
\hline \begin{tabular}{l|l}
48 & KINLOCH
\end{tabular} & Jan. 03, 1999 & -31 & -23 & 0 & 5 & 10 & 15 & clear & mostly clear \\
\hline 49 KUTAWAGAN & Dec. 27,1998 & -19 & -15 & 10 & 20 & 0 & 2 & partly cloudy & overcast \\
\hline
\end{tabular}




\begin{tabular}{|c|c|c|c|c|c|c|c|c|c|}
\hline COUNT & DATE & 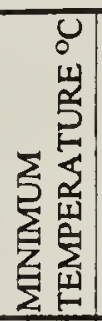 & 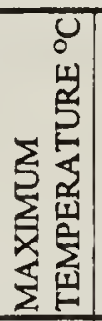 & 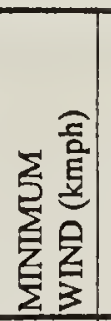 & 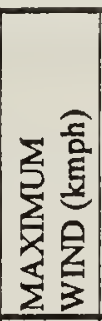 & 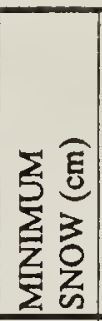 & 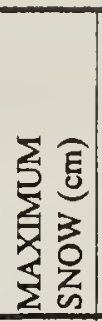 & SKY AM & SKY PM \\
\hline 50 LAST MTN. L. & Dec. 26, 1998 & -24 & -16 & 25 & 30 & 0 & 0 & overcast & overcast \\
\hline 51 LEADER (N) & Dec. 26,1998 & -16 & -8 & 10 & 20 & 0 & 20 & partly cloudy & partly cloudy \\
\hline 52 LEADER (S) & Dec. 26,1998 & -20 & -10 & 15 & 17 & 5 & 8 & clear & clear \\
\hline 53 LIVELONG & Dec. 30,1998 & -20 & -15 & 0 & 0 & 20 & 25 & overcast & partly cloudy \\
\hline 54 LOVE-TORCH R. & Dec. 26, 1998 & -23 & -21 & 0 & 5 & 20 & 30 & overcast & overcast \\
\hline 55 LUSELAND & Dec. 26,1998 & -14 & -8 & 10 & 15 & 0 & 4 & partly cloudy & partly cloudy \\
\hline 56 MACDOWALL & Dec. 25,1998 & -21 & -18 & 0 & 6 & 1 & 3 & mostly clear & partly cloudy \\
\hline 57 MAYVIEW & Dec. 23,1998 & -21 & -21 & 20 & 20 & 15 & 15 & partly cloudy & partly cloudy \\
\hline 58 MEADOW LAKE & Dec. 27,1998 & -18 & -15 & 0 & 5 & 12 & 15 & overcast & overcast \\
\hline 59 MELFORT & Dec. 28,1998 & -32 & -28 & 0 & 5 & 0 & 5 & partly cloudy & partly cloudy \\
\hline 60 MELVILLE & Dec. 26,1998 & -23 & -18 & 10 & 10 & 5 & 30 & clear & clear \\
\hline 61 MISSINIPE & Jan. 01,1999 & -32 & -30 & 0 & 0 & 10 & 20 & overcast & overcast \\
\hline 62 MOOSE JAW & Dec. 26,1998 & -14 & -7 & 11 & 19 & 0 & 2 & partly cloudy & overcast \\
\hline $63 \mathrm{MOOSE}$ & ,Dec. 30, 1998 & -18 & -13 & 10 & 30 & 6 & 13 & overcast & overcast \\
\hline 64 NAICAM & Dec. 31,1998 & -18 & -12 & 0 & 5 & 25 & 30 & partly cloudy & overcast \\
\hline 65 NIPAWIN & Dec. 29,1998 & -30 & -30 & 0 & 5 & 8 & 10 & & clear \\
\hline 66 NISBET FOREST & Jan. 01, 1999 & -20 & -15 & 0 & 8 & 1 & 15 & partly cloudy & mostly clear \\
\hline 67 PIKE LAKE & Jan. 02,1998 & -26 & -23 & 0 & 10 & 10 & 25 & clear & clear \\
\hline 68 PORCUPINE & Dec. 28,1998 & -28 & -20 & 5 & 10 & 15 & 25 & clear & clear \\
\hline 69 PREECEVILLE & Dec. 27,1998 & -15 & -11 & 15 & 25 & 10 & 15 & overcast & partly cloudy \\
\hline 70 PRINCE ALBERT & Dec. 20,1998 & -34 & -27 & 10 & 15 & 8 & 15 & clear & mostly clear \\
\hline 71 PRINCE ALBERT & Dec. 20, 1998 & -34 & -25 & 0 & 10 & 10 & 20 & mostly clear & partly cloudy \\
\hline 72 QU'APPELLE V. & Dec. 20,1998 & -25 & -23 & 5 & 15 & 5 & 20 & mostly clear & mostly clear \\
\hline 73 RAYMORE & Dec. 25,1998 & -27 & -16 & 15 & 50 & 0 & 5 & clear & clear \\
\hline 74 REGINA & Dec. 26,1998 & -22 & -8 & 0 & 10 & 0 & 4 & mostly clear & overcast \\
\hline 75 ROCKGLEN & Jan. 02, 1999 & -18 & -16 & 15 & 20 & 25 & 30 & clear & clear \\
\hline 76 ROUND LAKE & Dec. 19,1998 & -27 & -16 & 0 & 15 & 6 & 8 & mostly clear & mostly clear \\
\hline 77 SK. LANDING & Dec. 22,1998 & -22 & -16 & 20 & 30 & 0 & 6 & overcast & mostly clear \\
\hline 78 SK. RIVER & Dec. 23, 1998 & -23 & -22 & 15 & 25 & 5 & 10 & clear & clear \\
\hline 79 SASKATOON & Dec. 26,1998 & -21 & -12 & 0 & 15 & 2 & 10 & overcast & partly cloudy \\
\hline 80 SHAMROCK & Dec. 22,1998 & -18 & -16 & 40 & 55 & 2 & 6 & clear & clear \\
\hline 81 SKULL CREEK & Dec. 26, 1998 & & & & & & & & \\
\hline 82 SNOWDEN & Dec. 30,1998 & -25 & -16 & 0 & 0 & 4 & 15 & overcast & clear \\
\hline 83 SPALDING & Dec. 27,1998 & -24 & -18 & 0 & 0 & 16 & 20 & clear & clear \\
\hline 84 SPRUCE HOME & Dec. 27,1998 & -30 & -10 & 0 & 0 & 5 & 10 & mostly clear & mostly clear \\
\hline 85 SQUAW RAPIDS & Jan. 02, 1999 & -30 & -27 & 10 & 20 & 20 & 30 & overcast & partly cloudy \\
\hline 86 SWIFT CURRENT & Jan. 03, 1999 & -21 & -18 & 10 & 20 & 15 & 30 & partly cloudy & partly cloudy \\
\hline \begin{tabular}{l|l}
87 & TISDALE
\end{tabular} & Jan. 02, 1999 & & & & & & & & \\
\hline \begin{tabular}{l|l}
88 & TOGO
\end{tabular} & Dec. 27,1998 & -28 & -28 & 0 & 0 & 15 & 15 & & \\
\hline 89 TURTLE LAKE & Jan. 01, 1999 & -30 & -20 & 0 & 0 & 12 & 12 & & \\
\hline 90 WALDHEIM & Dec. 19,1998 & -25 & -20 & & & 5 & 5 & & \\
\hline 91 WATSON & Jan. 03, 1999 & -30 & -27 & 0 & 0 & 8 & 25 & clear & clear \\
\hline 92 WELDON - 1 & Jan. 01, 1998 & -30 & -25 & 0 & 5 & 10 & 12 & overcast & partly cloudy \\
\hline 93 WELDON - 2 & Dec. 28,1998 & -25 & -20 & 0 & 5 & 8 & 15 & clear & clear \\
\hline 94 WEYBURN & Dec. 19,1998 & -23 & -18 & 0 & 10 & 5 & 7 & mostly clear & mostly clear \\
\hline 95 WHITE BEAR & Dec. 27,1998 & -12 & -10 & 0 & 0 & 0 & 15 & partly cloudy & mostly clear \\
\hline \begin{tabular}{l|l}
96 & WHITEWOOD \\
\end{tabular} & Dec. 27,1998 & -17 & -13 & 10 & 15 & 10 & 20 & mostly clear & mostly clear \\
\hline \begin{tabular}{l|l}
97 & YORKTON \\
\end{tabular} & Dec. 28,1998 & -28 & -24 & 5 & 15 & 5 & 20 & mostly clear & mostly clear \\
\hline
\end{tabular}




\begin{tabular}{|c|c|c|c|c|c|c|c|c|c|c|c|c|c|c|c|c|c|c|c|c|}
\hline \multirow[b]{2}{*}{ LOCALITY } & \multicolumn{6}{|c|}{ EFFORT } & \multicolumn{14}{|c|}{ HABITAT } \\
\hline & 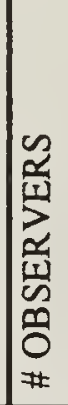 & $\begin{array}{l}5 \\
8 \\
\text { 岃 } \\
z \\
\text { z } \\
\vdots\end{array}$ & $\begin{array}{l}8 \\
8 \\
11 \\
2 \\
0 \\
2 \\
5 \\
5 \\
0\end{array}$ & 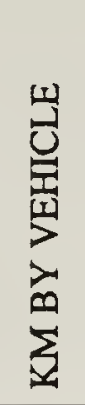 & 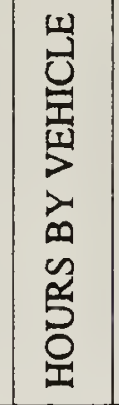 & 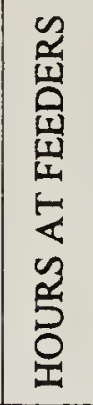 & 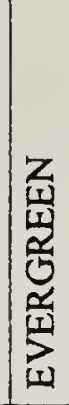 & 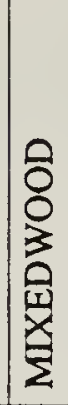 & 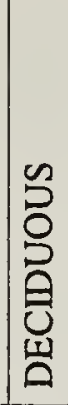 & 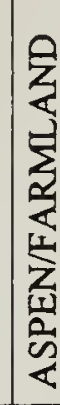 & $\begin{array}{l}5 \\
E \\
E \\
6 \\
6\end{array}$ & 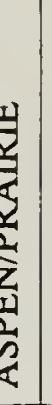 & 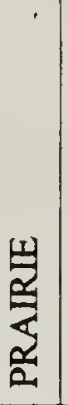 & 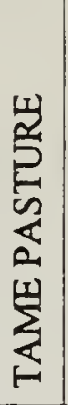 & 再 & 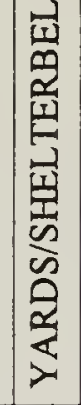 & 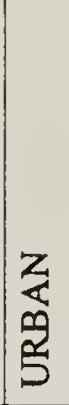 & 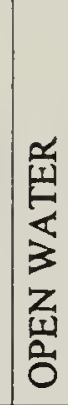 & 量 & $\mid \begin{array}{l}z \\
z \\
z\end{array}$ \\
\hline ARCHERWILL & 6 & 4 & 2 & 20 & 2 & 6 & & 10 & 15 & & & & & & & 60 & 15 & & & \\
\hline ARMIT & 3 & 10 & 4 & 105 & 4 & & 10 & 40 & 40 & 10 & & & & & & & & & & \\
\hline BANGOR & 1 & 2 & 1 & 10 & 2 & 4 & & & & 25 & & & & & 25 & 50 & & & & \\
\hline BEAUVAL & 2 & & & & & & 25 & 60 & 10 & & & & & 2 & 1 & 2 & & & & \\
\hline BIGGAR & 5 & 23.5 & 10.75 & 245 & 9.75 & 2 & & & & 15 & & & & & 27 & 28 & 30 & & & \\
\hline BIRCH HILLS & 3 & 2 & 1 & 98 & 4.5 & 3 & & & 4 & 30 & & 5 & & 5 & 40 & 10 & 5 & 1 & & \\
\hline BRIGHTW ATER & 2 & 5 & 2.5 & 114 & 5.75 & & & & 15 & & & 20 & 10 & 4 & 20 & 30 & & 1 & & \\
\hline BROADVIEW & 7 & 4 & 1.5 & 160 & 5.5 & 2 & 1 & & 14 & 20 & & & 15 & & 30 & 10 & 10 & & & \\
\hline BROMHEAD & 2 & 1 & 0.5 & 59 & 4.5 & & & & & & & & & & 90 & 10 & & & & \\
\hline CANDLE LAKE & 4 & 4 & 2 & 158 & 4.5 & & 20 & 50 & 20 & & & & & & & & 10 & & & \\
\hline CHITEK LAKE & 1 & 3 & 2 & 15 & 2.5 & 0.5 & 10 & 10 & & 25 & & & & & & & 50 & & 5 & \\
\hline CLARK'S CROSS. & 29 & 31.6 & 20.9 & 525 & 26.6 & 7 & 1 & & 2 & 12 & & 0 & 3 & 2 & 11 & 14 & 19 & 2 & 4 & 18 \\
\hline COCHIN & 3 & 2 & 0.75 & 90 & 5 & & & & 10 & 60 & & 5 & & & 20 & & 5 & & & \\
\hline COLD L.-PIERCEL. & 2 & 2 & 1.5 & 131 & 5 & 0.5 & & 30 & & & & & & & 10 & 10 & 50 & & & \\
\hline CORONACH & 3 & 1 & 1 & 180 & 7 & & & & & & & & 10 & & 60 & 5 & 5 & 20 & & \\
\hline CRAVEN & 11 & 5 & 3 & 377 & 19 & 2 & & & & 10 & & 5 & & & 35 & 10 & 25 & 5 & & \\
\hline CROOKED LAKE & 6 & 0.2 & 0.25 & 166 & 5.5 & 1.25 & & & 25 & 15 & & & & & & 5 & 15 & & 40 & \\
\hline CROOKED RIVER & 2 & 1 & 1 & 50 & 1 & 2.5 & & & 33 & & & 33 & & & & 34 & & & & \\
\hline CYPRESS HILLS & 11 & 15 & 4 & 30 & 4 & 3 & 60 & 20 & & & & & 10 & & & 10 & & & & \\
\hline DUCK LAKE & 2 & 10 & 4 & 105 & 4.5 & & & 65 & 5 & 15 & & & & & 4 & 1 & 10 & & & \\
\hline DUVAL & 2 & 3 & 2 & 131 & 4 & 1 & & & 10 & 5 & & 1 & 1 & & 40 & 23 & 20 & & & \\
\hline EASTEND & 2 & 4 & 3.25 & 40 & 3 & & & & & & & & 15 & & 30 & & 55 & & & \\
\hline EMMA LAKE & 4 & 1.5 & 1 & 55 & 3.5 & 4 & & 95 & & & & & & & & 5 & & & & \\
\hline ENDEAVOUR & 1 & 4 & 3 & 20 & 2 & & & 40 & & 60 & & & & & & & & & & \\
\hline ESTEVAN & 3 & 5 & 3 & 145 & 7 & & & & 20 & & & & 20 & & 20 & & 20 & 20 & & \\
\hline ESTUARY & 2 & 5 & 3 & 120 & 3 & & & & 25 & & & & 45 & & & 30 & & & & \\
\hline FENTON & 2 & 2 & 0.75 & 130 & 4.75 & 1 & & & & 10 & & & & & 75 & 10 & 5 & & & \\
\hline FIFE LAKE & 2 & 1 & 0.75 & 100 & 4 & & & & & & & & 5 & & 30 & 20 & 5 & 40 & & \\
\hline FORT QU'APPELLE & 16 & 1 & 1 & 60 & 3 & 11 & & 5 & 20 & & & 20 & 5 & & 10 & 10 & 20 & 10 & & \\
\hline FORT WALSH & 10 & 50 & 24.5 & 123 & 11.5 & & & 33 & & & & & 2 & & 4 & 4 & & & & 5 \\
\hline GARDINER DAM & 15 & 12.5 & 6.25 & 309 & 29.25 & & & & & 7 & 7 & & & & 32 & 31 & & 20 & & 1 \\
\hline GOOD SPIRIT LAKE & 5 & 1 & 0.5 & 108 & 5.5 & 0.5 & & 2 & 3 & 5 & 52 & 20 & 40 & 2 & 5 & 20 & 2 & 1 & & \\
\hline GOVENLOCK & 9 & 16 & 8 & 300 & 13 & & & & & & & & 50 & & 25 & 25 & & & & \\
\hline GRASSLANDS N.P. & 9 & 3 & 1 & 150 & 8 & & & & & & & & 80 & 10 & 5 & 5 & & & & \\
\hline GRAYSON & 4 & 7 & 2 & 50 & 3 & 1 & & & 20 & 30 & & & & & 15 & 20 & 5 & 10 & & \\
\hline HARRIS & 3 & 8 & 4 & 173 & 6.5 & & & & & 19 & & & & & 32 & 32 & 17 & & & \\
\hline HEPBURN & 2 & 1 & 1 & 12 & 1 & 3 & & & & 10 & & & & & & & 90 & & & \\
\hline HERSCHAL & 2 & 6 & 2.5 & 225 & 4 & & & & & 45 & 51 & 0 & 5 & & & 5 & & & & 3 \\
\hline HOLBEIN & 2 & & & & & 8 & & & & & & & & & & 100 & & & & \\
\hline HORSESHOE BEND & 4 & 1 & 3 & & & 1 & & 10 & & & & 0 & & & & 80 & & & & \\
\hline INDIAN HEAD & 15 & 8 & 2 & 193 & 4 & 15 & & & & 20 & & 0 & & 5 & 20 & 20 & 20 & 5 & & \\
\hline KAMSACK & 21 & & & & & & & & & & & & & & & 100 & & & & \\
\hline KELVINGTON & 3 & & & 62 & 2.5 & 5 & & & & 30 & & & & & 60 & 10 & & & & \\
\hline KENASTON & 1 & 1 & 0.5 & 15 & 2 & 2 & & & & 40 & & & & & 10 & 45 & 5 & & & \\
\hline KENOSEE LAKE & 2 & & & 108 & 3.5 & 1 & & & 20 & 20 & & & & & & & 60 & & & \\
\hline KINDERSLEY & 2 & & & 87 & 4 & & & & & & & & & 8 & 30 & 60 & 2 & & & \\
\hline KINISTINO & 1 & 4 & 2 & 21 & 0.5 & 1 & & & & & & & & & & 70 & 30 & & & \\
\hline KINLOCH & 6 & 3 & 2 & 45 & 1.5 & 13 & 15 & 20 & 15 & 10 & & & & & & 40 & & & & \\
\hline
\end{tabular}


Table 2-2: COUNT COVERAGE

\begin{tabular}{|c|c|c|c|c|c|c|c|c|c|c|c|c|c|c|c|c|c|c|c|c|c|}
\hline \multirow[b]{2}{*}{ LOCALITY } & \multicolumn{6}{|c|}{ EFFORT } & \multicolumn{15}{|c|}{ HABITAT } \\
\hline & 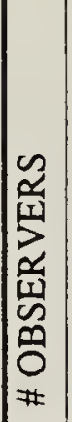 & $\begin{array}{l}5 \\
8 \\
z \\
z \\
z \\
z\end{array}$ & 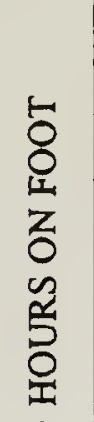 & 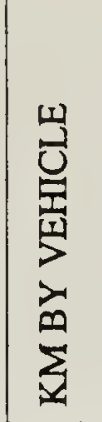 & 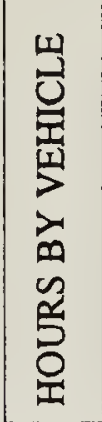 & 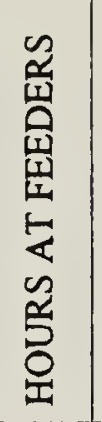 & 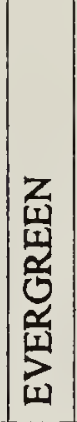 & 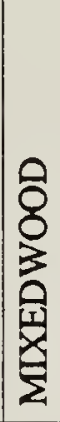 & 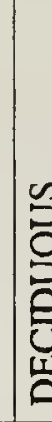 & & 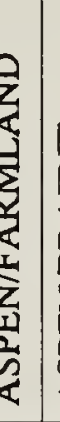 & 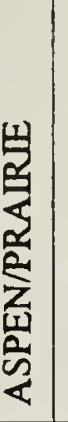 & $\begin{array}{l}\text { 岁 } \\
\text { 占 } \\
\text { 吕 }\end{array}$ & 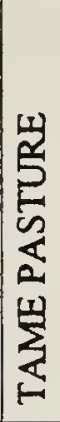 & 獣 & 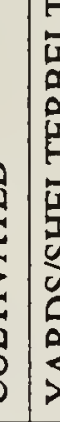 & 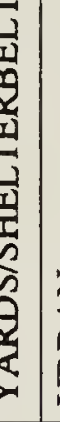 & $\begin{array}{l}z \\
x \\
z \\
5\end{array}$ & 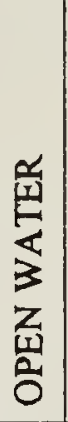 & 岂 & 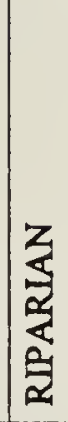 \\
\hline KUTAWAGAN & 3 & 2 & 1 & 210 & 8 & & & & & & 20 & 10 & 10 & & & 10 & 10 & 10 & & & \\
\hline LAST MTN. L. & 4 & 3 & 2 & 210 & 5 & & & & & & 10 & & 30 & & & 30 & 5 & 4 & 1 & & \\
\hline LEADER (S) & 2 & & & 270 & 6 & 3 & & & & & & & 50 & & 3 & \begin{tabular}{l|l}
0 & 1 \\
\end{tabular} & 10 & 10 & & & \\
\hline LIVELONG & 1 & 4 & 2 & & & 4 & & & & & & & & & 2 & 0 & & 80 & & & \\
\hline LOVE-TORCH R. & 17 & 2 & 2 & 100 & 4 & 28 & & 10 & & & 10 & & & & 1 & \begin{tabular}{l|l}
0 & 5 \\
\end{tabular} & 50 & 20 & & & \\
\hline LUSELAND & 10 & 14 & 6 & 250 & 5 & 4 & & & & & 20 & 10 & 5 & 5 & 1 & \begin{tabular}{l|l}
52 \\
\end{tabular} & 20 & 25 & & & \\
\hline MACDOWALL & 1 & 2 & 2 & 25 & 2 & 2 & 10 & 10 & 1 & & 50 & & & & 1 & \begin{tabular}{l|l}
0 & 1 \\
\end{tabular} & 10 & & & & \\
\hline MAYVIEW & 1 & & & & & & & 99 & & & & & & & & & 1 & & & & \\
\hline MEADOW LAKE & 7 & 9 & 4.5 & 135 & 4.5 & 8 & & 20 & & & & & & & 1 & $\begin{array}{l}5 \sqrt[3]{3} \\
\end{array}$ & 30 & 35 & & & \\
\hline MELFORT & 1 & 2 & 1 & & & 2 & & & & & & & & & 2. & 57 & 75 & & & & \\
\hline MELVILLE & 4 & 3 & 2 & 25 & 1.5 & 3 & & & & & 10 & & & & & 56 & 60 & 25 & & & \\
\hline MISSINIPE & 1 & 3 & 2 & & & 3 & & 28 & & & & & & & & & & 72 & & & \\
\hline MOOSE JAW & 28 & 13 & 7 & 82 & 4 & 7 & & & & & & & & & 1 & 0 & & 45 & & & 45 \\
\hline MOOSE MOUNTAIN & 3 & 4 & 1 & 182 & 4.5 & & & & 4 & & 20 & & & & 1 & 5 & & 25 & & & \\
\hline NAICAM & 1 & & & & & 6 & & & & & & & & & & & 00 & & & & \\
\hline NIPAWIN & 9 & & & & 3 & 3 & & 80 & & & 10 & & & & & & & 10 & & & \\
\hline NISBET FOREST & 4 & 5 & 1 & 100 & 4 & & 10 & & 3 & & & & & & 4 & \begin{tabular}{l|l}
0 & 1 \\
0
\end{tabular} & 10 & 10 & & & \\
\hline PIKE LAKE & 37 & 46 & 20 & 334 & 12 & 4.9 & & & 1 & & 10 & & 5 & & 1 . & \begin{tabular}{l|l}
5 & 1 \\
\end{tabular} & 15 & 40 & & & \\
\hline PORCUPINE PLAIN & 1 & 1 & 1 & 60 & 4.5 & 1 & & & & & & & & & & & & & & & \\
\hline PREECEVILLE & 9 & 9 & 6 & 142 & 5 & 16 & & 25 & & & 50 & 20 & & & & & 5 & & & & \\
\hline PRINCE ALBERT & 24 & 19.5 & 4.3 & 560 & 20.8 & 25 & & 20 & & & & & & & & 5 & & 75 & & & \\
\hline PRINCE ALBERT & 21 & 35 & 19.5 & 69 & 3.5 & & 20 & 50 & 2 & & & & & & & & & 5 & 5 & & \\
\hline QUAPPELLE V. & 4 & 4 & 3.6 & 211 & 5.1 & & & 1 & 1 & 5 & 10 & 5 & 4 & 15 & 2 & \begin{tabular}{l|l}
0 & 2 \\
\end{tabular} & 20 & 5 & 5 & & \\
\hline RAYMORE & 4 & 5 & 3 & 231 & 7 & 1 & & & & & 70 & & & & 1 & \begin{tabular}{l|l}
0 & 1 \\
\end{tabular} & 10 & 10 & & & \\
\hline REGINA & 30 & 69 & 28 & 741 & 26 & 3 & & & & 8 & 3 & 5 & 6 & & 3 & \begin{tabular}{l|l}
0 & 2 \\
0
\end{tabular} & 22 & 26 & & & \\
\hline ROCKGLEN & 1 & 1 & 1 & 80 & 4 & & & & & & & & 20 & & 7 & \begin{tabular}{l|l}
0 & 1 \\
\end{tabular} & 10 & & & & \\
\hline ROUND LAKE & 5 & 1 & 0.5 & 228 & 10 & & & & & & 40 & 10 & & & 2. & 5 & 5 & & 20 & & \\
\hline SK. LANDING P.P. & 6 & 5 & 3 & 150 & 10 & & & & & & & & 30 & & 3 & 0 & 9 & & 1 & & 30 \\
\hline SK. RIVER FORKS & 2 & 2.5 & 1.5 & 75 & 3.5 & & 10 & 20 & & 5 & 5 & & & 5 & 4 & 0.1 & 10 & & 5 & & \\
\hline SASKATOON & 101 & 98 & 60 & 1052 & 63 & 97 & & 1 & & 4 & 12 & & 1 & 1 & & $9[1$ & 12 & 58 & 2 & & \\
\hline SHAMROCK & 1 & & & 64 & 3.5 & & & & & & & & & 5 & 8 & \begin{tabular}{c|c}
5 & 1 \\
\end{tabular} & 10 & & & & \\
\hline SKULL CREEK & 13 & & & & & & & & & & & & & & & & & & & & \\
\hline SNOWDEN & 18 & 2.5 & 1.5 & 126 & 3.5 & 4 & & 10 & & & & & & & 2 & 055 & 50 & 20 & & & \\
\hline SPALDING & 5 & 1 & 0.5 & 45 & 3 & 3 & & 40 & & & & & & & & & 20 & 40 & & & \\
\hline SPRUCE HOME & 2 & & & & & 3 & & 50 & & & 50 & & & & & & & & & & \\
\hline SQUAW RAPIDS & 4 & 20 & 8 & 130 & 8 & & 10 & 60 & 1 & & & 10 & & & & & & & 10 & & \\
\hline SWIFT CURRENT & 22 & 24 & 12 & 346 & 15.5 & 17.5 & & & & & & & 2 & 2 & 8 & 0 & 5 & 10 & 1 & & \\
\hline TISDALE & 7 & & & & & 12 & & & & & & & & & 3 & $\begin{array}{ll}0 & 7 \\
0\end{array}$ & 70 & & & & \\
\hline TOGO & 2 & & & & & 3 & & & & & & 00 & & & & & & & & & \\
\hline TURTLE LAKE & 2 & & & 106 & 5 & 6 & 25 & 25 & & & 25 & & & & 2 & 5 & & & & & \\
\hline WALDHEIM & 2 & & & 50 & 1 & 4 & & & & & & & & & 4 & 0 & & 60 & & & \\
\hline WATSON & 5 & 1 & 1 & 73 & 3.75 & 2 & & & 2 & & & 25 & & & 2 & \begin{tabular}{l|l}
5 & 1 \\
\end{tabular} & 15 & 10 & & & \\
\hline WELDON - 1 & 2 & & & & & 7 & & & & & & 40 & & & & & 60 & & & & \\
\hline WELDON - 2 & 1 & 3 & 2 & 55 & 2 & 1. & & 50 & & & 25 & & & & & & 25 & & & & \\
\hline WEYBURN & 13 & 7 & 3 & 295 & 4 & 1 & & & & & & & 5 & & 5 & \begin{tabular}{l|l}
0 & 1 \\
\end{tabular} & 10 & 35 & & & \\
\hline WHITE BEAR & 6 & 22 & 8 & 48 & 1.5 & & & & & & & & & & 1 & 5 & 5 & & & & 80 \\
\hline NOOD & 21 & 12 & 8.5 & 381 & 11.5 & 24 & & & & & 25 & & & & & \begin{tabular}{l|l}
5 & 2 \\
\end{tabular} & 20 & 50 & & & \\
\hline YORKTON & 11 & 5 & 2 & 151 & 8 & 1 & & & & & 50 & & & & & & & 50 & & & \\
\hline
\end{tabular}


Table 3-1. SPECIES RECORDED FROM MORE THAN EIGHT LOCALITIES

$(+=$ species seen during the count period but not on count day)

SPECIES

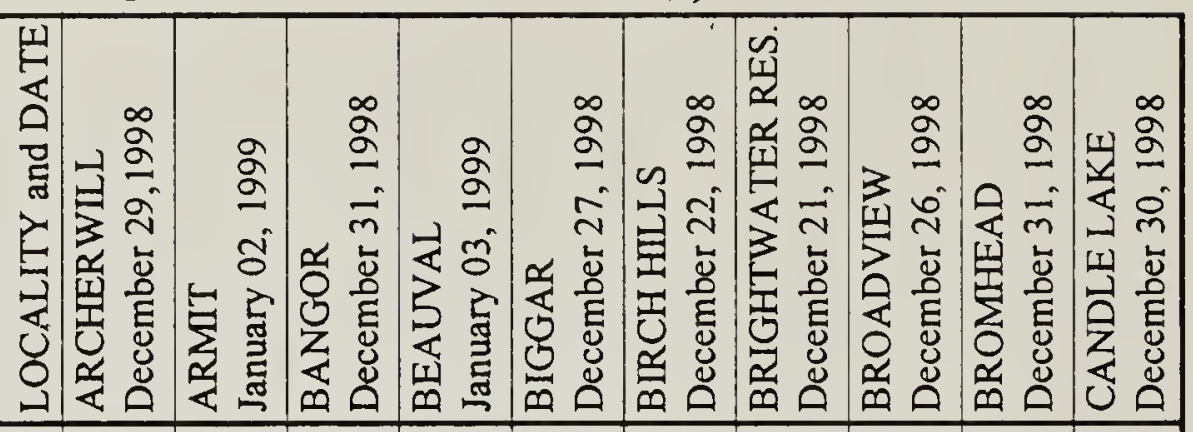

CANADA GOOSE

MALLARD

COMMON GOLDENEYE

BALD EAGLE

NORTHERN GOSHAWK

GOLDEN EAGLE

MERLIN

GRAY PARTRIDGE

RING-NECKED PHEASANT

RUFFED GROUSE

SHARP-TAILED GROUSE

ROCK DOVE

GREAT HORNED OWL

SNOWY OWL

SHORT-EARED OWL

DOWNY WOODPECKER

HAIRY WOODPECKER

BLACK-BACKED WOODPECKER

NORTHERN FLICKER (Y-s)

PILEATED WOODPECKER

HORNED LARK

GRAY JAY

BLUE JAY

BLACK-BILLED MAGPIE

COMMON RAVEN

BLACK-CAPPED CHICKADEE

BOREAL CHICKADEE

RED-BREASTED NUTHATCH

WHITE-BREASTED NUTHATCH

GOLDEN-CROWNED KINGLET

AMERICAN ROBIN

BOHEMIAN WAXWING

NORTHERN SHRIKE

EUROPEAN STARLING

WHITE-THROATED SPARROW

DARK-EYED JUNCO (S-c)

SNOW BUNTING

PINE GROSBEAK

HOUSE FINCH

RED CROSSBILL

COMMON REDPOLL

HOARY REDPOLL

PINE SISKIN

EVENING GROSBEAK

HOUSE SPARROW

INDIVIDUALSINTABLES 4 \& 5

SPECIES IN TABLES 4 \& 5

INDIVIDUALS ON COUNT DAY

SPECIES ON COUNT DAY

SPECIES COUNT PERIOD

\begin{tabular}{|c|c|c|c|c|c|c|c|c|c|}
\hline & & & & & & 114 & & & \\
\hline & & & & & & & & & \\
\hline \multicolumn{10}{|l|}{2} \\
\hline & & & 1 & 1 & & & & & 1 \\
\hline & & & & & & 1 & & 1 & \\
\hline & & & & 1 & & & + & & \\
\hline & & & & 12 & 39 & 14 & & & \\
\hline & & & & & & & & 42 & \\
\hline 1 & 6 & + & 2 & & 1 & & 2 & & \\
\hline \multirow[t]{5}{*}{3} & 17 & + & & 75 & & 34 & 26 & 2 & 3 \\
\hline & & 5 & & 104 & 92 & 32 & 8 & 16 & \\
\hline & & & + & 6 & 1 & & 1 & 1 & \\
\hline & & & & 1 & & & & 2 & \\
\hline & & & & & & & & 1 & \\
\hline 6 & 2 & 2 & 1 & 5 & 4 & 1 & 1 & & 1 \\
\hline \multirow[t]{5}{*}{5} & 7 & 3 & 4 & 3 & 8 & 1 & 5 & & 1 \\
\hline & 1 & & & & & & & & 1 \\
\hline & & & & & & & & & \\
\hline & & & & & & & & & \\
\hline & & & & & & 18 & & 805 & \\
\hline 1 & 13 & & 7 & & & & & & 9 \\
\hline 28 & 8 & 2 & 10 & 2 & 1 & 2 & & & 2 \\
\hline 11 & 37 & 3 & 57 & 202 & 53 & 40 & 45 & 5 & 24 \\
\hline 8 & 27 & 2 & 50 & 15 & 24 & 7 & 49 & & 137 \\
\hline \multirow[t]{2}{*}{79} & 24 & 15 & 10 & 43 & 36 & 4 & 26 & & 19 \\
\hline & 4 & & 1 & 2 & & & & & 10 \\
\hline \multirow[t]{9}{*}{8} & 1 & & & 5 & & & & & \\
\hline & & & & & & & 3 & & \\
\hline & & & & 3 & & & & & \\
\hline & & & & 2 & & & & & \\
\hline & & + & & 381 & 13 & & 200 & & \\
\hline & & & & 1 & & & & & \\
\hline & & & & 3 & & & & & \\
\hline & & & & & 1 & & & & \\
\hline & & + & & 2 & 1 & & & & \\
\hline 37 & 5061 & 50 & & 1231 & 346 & 2 & 1 & 3112 & \\
\hline \multirow[t]{3}{*}{43} & & 2 & 64 & 3 & & & & & 8 \\
\hline & & & & 1 & & & & & \\
\hline & & 15 & & 3 & & & & & \\
\hline \multirow[t]{3}{*}{27} & 60 & 9 & 22 & 645 & 13 & & 1 & & \\
\hline & 3 & & 3 & 7 & & & & & \\
\hline & & & & 5 & & & & & \\
\hline 55 & 11 & & 60 & & & & & & \\
\hline 58 & 2 & & & 881 & 124 & 184 & 122 & 28 & \\
\hline 1 & 5 & 0 & 2 & 2 & 0 & 0 & 3 & 1298 & 2 \\
\hline 2 & 4 & 1 & 2 & 2 & 0 & 0 & 1 & 1 & 1 \\
\hline 373 & 5289 & 108 & 294 & 3647 & 757 & 454 & 493 & 5313 & 218 \\
\hline 17 & 21 & 11 & 16 & 31 & 16 & 14 & 15 & 12 & 13 \\
\hline 18 & 21 & 16 & 17 & 31 & 16 & 14 & 16 & 12 & 13 \\
\hline
\end{tabular}


Table 3-2. SPECIES RECORDED FROM MORE THAN EIGHT LOCALITIES ( $+=$ species seen during the count period but not on count day)

\begin{tabular}{|c|c|c|c|c|c|c|c|c|c|c|}
\hline \multirow[t]{16}{*}{ 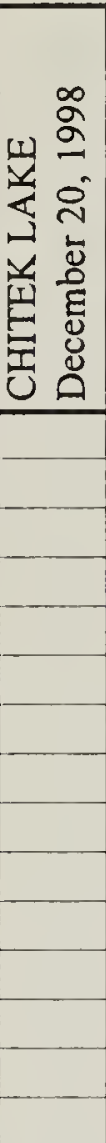 } & 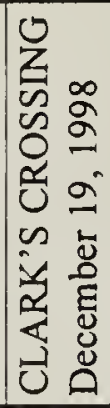 & 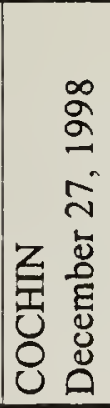 & 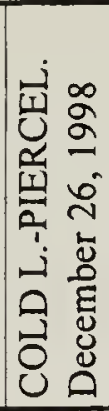 & 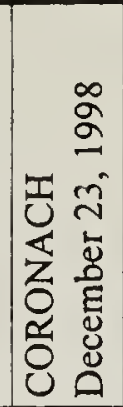 & 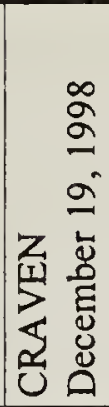 & 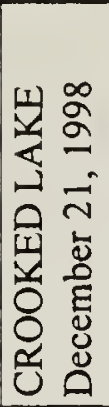 & 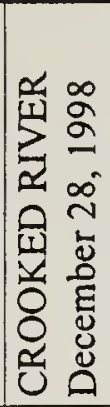 & 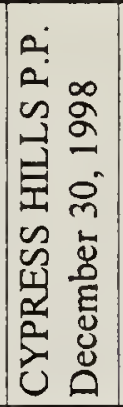 & 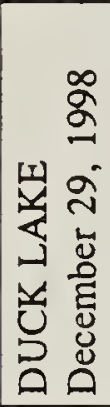 & 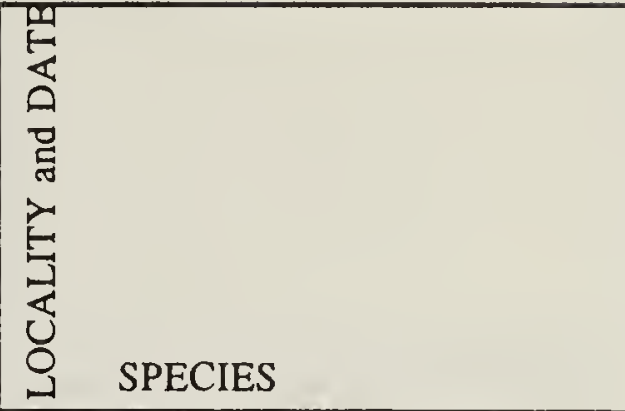 \\
\hline & 125 & & & 492 & & & & & & \multirow{5}{*}{$\begin{array}{l}\text { CANADA GOOSE } \\
\text { MALLARD } \\
\text { COMMON GOLDENEYE } \\
\text { BALD EAGLE } \\
\text { NORTHERN GOSHAWK }\end{array}$} \\
\hline & & & & 13700 & & 263 & & & & \\
\hline & 1 & & & 31 & 17 & 10 & & & & \\
\hline & 1 & & & 1 & & 1 & & & & \\
\hline & & & & & & & & 1 & & \\
\hline & & 1 & & 2 & 1 & & & & & \multirow{5}{*}{$\begin{array}{l}\text { GOLDEN EAGLE } \\
\text { MERLIN } \\
\text { GRAY PARTRIDGE } \\
\text { RING-NECKED PHEASANT } \\
\text { RUFFED GROUSE }\end{array}$} \\
\hline & 1 & & & & 1 & & & & & \\
\hline & 212 & 27 & & & & & & & & \\
\hline & & & & 11 & & & & 1 & & \\
\hline & 1 & 2 & & & + & & 2 & & 1 & \\
\hline & 43 & 5 & & 2 & 14 & & 4 & & 1 & \multirow{5}{*}{$\begin{array}{l}\text { SHARP-TAILED GROUSE } \\
\text { ROCK DOVE } \\
\text { GREAT HORNED OWL } \\
\text { SNOWY OWL } \\
\text { SHORT-EARED OWL }\end{array}$} \\
\hline & 178 & 6 & 7 & 24 & 54 & 22 & & & 10 & \\
\hline & 1 & 1 & & & 1 & 1 & & 1 & & \\
\hline & 4 & & & & 2 & & + & & & \\
\hline & & & & & & & & & & \\
\hline 3 & 6 & 1 & 1 & & 12 & 2 & 2 & 5 & 2 & DOWNY WOODPECKER \\
\hline 1 & 9 & & 2 & & 4 & 1 & 2 & 4 & 1 & \multirow{3}{*}{$\begin{array}{l}\text { BLACK-BACKED WOODPECKER } \\
\text { NORTHERN FLICKER (Y-s) }\end{array}$} \\
\hline \multirow[t]{4}{*}{1} & & & & & & & & & 1 & \\
\hline & & & & & & & & & & \\
\hline & & 1 & & & & & & & 1 & PILEATED WOODPECKER \\
\hline & & 17 & & 134 & & & & & & HORNED LARK \\
\hline 2 & & 2 & + & & & & & & 1 & GRAY JAY \\
\hline 3 & 1 & & 8 & & 12 & 13 & 6 & 1 & & BLUE JAY \\
\hline 14 & 339 & 12 & 44 & 27 & 98 & 49 & 20 & 59 & 13 & BLACK-BILLED MAGPIE \\
\hline 127 & 31 & 6 & 255 & & & 2 & 38 & 14 & 28 & COMMON RAVEN \\
\hline 20 & 71 & 3 & 29 & & 50 & 44 & 9 & 86 & 1 & \multirow{5}{*}{$\begin{array}{l}\text { BLACK-CAPPED CHICKADEE } \\
\text { BOREAL CHICKADEE } \\
\text { RED-BREASTED NUTHATCH } \\
\text { WHITE-BREASTED NUTHATCH } \\
\text { GOLDEN-CROWNED KINGLET }\end{array}$} \\
\hline \multirow[t]{11}{*}{5} & & & 2 & & & & & & & \\
\hline & & & 2 & & & & & 6 & & \\
\hline & & & & & 7 & 1 & & & & \\
\hline & & & 2 & & & & & & & \\
\hline & 1 & & & & + & & & & & \multirow{5}{*}{$\begin{array}{l}\text { AMERICAN ROBIN } \\
\text { BOHEMIAN WAXWING } \\
\text { NORTHERN SHRIKE } \\
\text { EUROPEAN STARLING } \\
\text { WHITE-THROATED SPARROW }\end{array}$} \\
\hline & 224 & 150 & & 16 & 584 & 16 & & 5 & & \\
\hline & & & & & 1 & 1 & + & & & \\
\hline & 50 & & 17 & 5 & & & & & & \\
\hline & 1 & & & & & 1 & & & & \\
\hline & 1 & & & & 2 & & 1 & 1 & & DARK-EYED JUNCO (S-c) \\
\hline & 1656 & 45 & & 40 & 26 & 150 & 50 & & 75 & \multirow{4}{*}{$\begin{array}{l}\text { SNOW BUNTING } \\
\text { PINE GROSBEAK } \\
\text { HOUSE FINCH } \\
\text { RED CROSSBILL }\end{array}$} \\
\hline \multirow[t]{3}{*}{21} & 4 & & 42 & & & & 34 & & & \\
\hline & 2 & & & & 6 & & & & & \\
\hline & & & & & & & & 22 & & \\
\hline \multirow[t]{3}{*}{8} & 267 & & 50 & & 7 & 680 & & & & \multirow{3}{*}{$\begin{array}{l}\text { COMMON REDPOLL } \\
\text { HOARY REDPOLL } \\
\text { PINE SISKIN }\end{array}$} \\
\hline & 17 & & & & & & & & & \\
\hline & & & & & 1 & & & & & \\
\hline \multirow[t]{2}{*}{5} & & & 2 & & & & 11 & 2 & 4 & EVENING GROSBEAK \\
\hline & 2156 & 13 & 54 & 558 & 393 & 371 & 23 & & 13 & HOUSE SPARROW \\
\hline 0 & $\mathrm{~T}$ & 0 & T9 & 57 & 5 & 2 & 0 & 2 & 2 & \multirow{2}{*}{ INDIVIDUALS IN TABLES 4 \& 5} \\
\hline 0 & 1 & 0 & 2 & 6 & 5 & 2 & 0 & 3 & 1 & \\
\hline 210 & 5404 & 292 & 536 & 15100 & 1298 & 1630 & 202 & 210 & 154 & INDIVIDUALS ON COUNT DAY \\
\hline 12 & 28 & 16 & 17 & 20 & 24 & 20 & 13 & 16 & 15 & SPECIES ON COUNT DAY \\
\hline 12 & 28 & 16 & 18 & 20 & 28 & 20 & 15 & 17 & 15 & SPECIES COUNT PERIOD \\
\hline
\end{tabular}


Table 3-3. SPECIES RECORDED FROM MORE THAN EIGHT LOCALITIES $(+=$ species seen during the count period but not on count day)

\begin{tabular}{|c|c|c|c|c|c|c|c|c|c|}
\hline 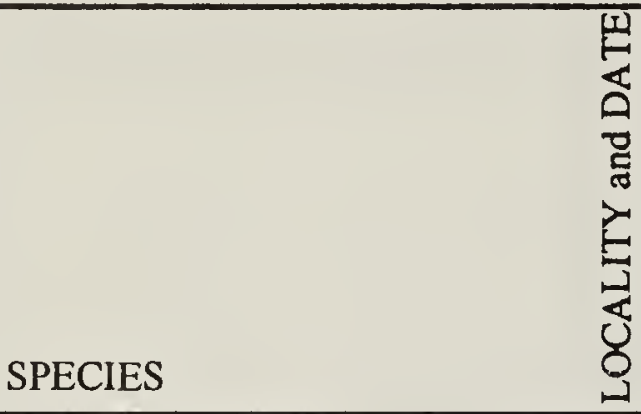 & 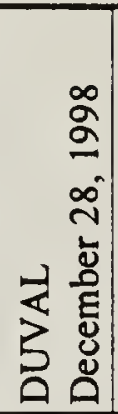 & 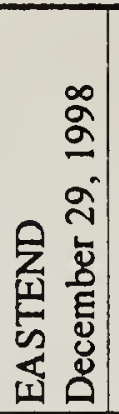 & 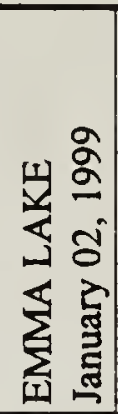 & 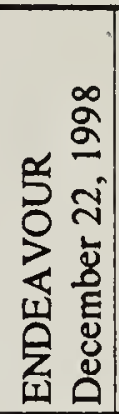 & 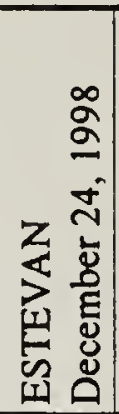 & 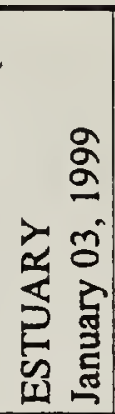 & 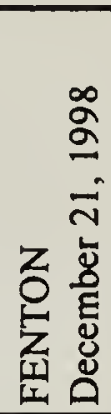 & 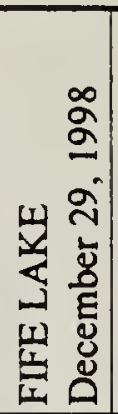 & 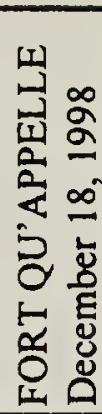 \\
\hline \multirow{5}{*}{$\begin{array}{l}\text { CANADA GOOSE } \\
\text { MALLARD } \\
\text { COMMON GOLDENEYE } \\
\text { BALD EAGLE } \\
\text { NORTHERN GOSHAWK } \\
\end{array}$} & & & & & 701 & & & 349 & 549 \\
\hline & & & & & 241 & & & 1543 & 95 \\
\hline & & & & & 2 & & & 36 & 31 \\
\hline & & & & & & & & 2 & 3 \\
\hline & & & & & & 1 & & & \\
\hline \multirow{5}{*}{$\begin{array}{l}\text { GOLDEN EAGLE } \\
\text { MERLIN } \\
\text { GRAY PARTRIDGE } \\
\text { RING-NECKED PHEASANT } \\
\text { RUFFED GROUSE } \\
\end{array}$} & + & + & & + & 1 & & & 3 & \\
\hline & & 1 & & & & & & & \\
\hline & 8 & & & & & 13 & & 33 & \\
\hline & & 4 & & & 11 & 7 & & & \\
\hline & 1 & & & 1 & & & 1 & & \\
\hline \multirow{5}{*}{$\begin{array}{l}\text { SHARP-TAILED GROUSE } \\
\text { ROCK DOVE } \\
\text { GREAT HORNED OWL } \\
\text { SNOWY OWL } \\
\text { SHORT-EARED OWL }\end{array}$} & & & & 11 & & 44 & & 34 & \\
\hline & 35 & & & + & 2 & 25 & 16 & 10 & \\
\hline & 3 & 2 & & + & & & 1 & & + \\
\hline & 1 & & & & & & & 2 & 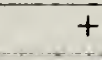 \\
\hline & & & & & & & & & \\
\hline \multirow{5}{*}{$\begin{array}{l}\text { DOWNY WOODPECKER } \\
\text { HAIRY WOODPECKER } \\
\text { BLACK-BACKED WOODPECKER } \\
\text { NORTHERN FLICKER }(Y-s) \\
\text { PILEATED WOODPECKER }\end{array}$} & 10 & 3 & 4 & 1 & 3 & & 5 & & 13 \\
\hline & 5 & 3. & 3 & 11 & 2 & 1 & 5 & & 11 \\
\hline & & & + & + & & & & & \\
\hline & & & & & & 2 & & & \\
\hline & & & & & & & & & \\
\hline \multirow{5}{*}{$\begin{array}{l}\text { HORNED LARK } \\
\text { GRAY JAY } \\
\text { BLUE JAY } \\
\text { BLACK-BILLED MAGPIE } \\
\text { COMMON RAVEN }\end{array}$} & 3 & 135 & & & & 185 & & 1596 & \\
\hline & & & 4 & & & & & & \\
\hline & 12 & 1 & 9 & 3 & & 9 & 2 & & 33 \\
\hline & 22 & 31 & 2 & 2 & 29 & 52 & 46 & 4 & 14 \\
\hline & 4 & & 124 & 80 & & & 16 & & \\
\hline \multirow{5}{*}{$\begin{array}{l}\text { BLACK-CAPPED CHICKADEE } \\
\text { BOREAL CHICKADEE } \\
\text { RED-BREASTED NUTHATCH } \\
\text { WHITE-BREASTED NUTHATCH } \\
\text { GOLDEN-CROWNED KINGLET }\end{array}$} & 31 & 20 & 99 & 9 & 35 & 13 & 21 & & 133 \\
\hline & & & 32 & & & & & & \\
\hline & & & 3 & & & & & & \\
\hline & & & 9 & 2 & & & & & 17 \\
\hline & & & & & & & & & \\
\hline \multicolumn{10}{|l|}{ AMERICAN ROBIN } \\
\hline \multirow{4}{*}{$\begin{array}{l}\text { BOHEMIAN WAXWING } \\
\text { NORTHERN SHRIKE } \\
\text { EUROPEAN STARLING } \\
\text { WHITE-THROATED SPARROW }\end{array}$} & 26 & 54 & & & 60 & 269 & 1 & & 604 \\
\hline & & 2 & & & 1 & & & & \\
\hline & 3 & & & & 18 & & & & \\
\hline & & & & & & & & & + \\
\hline \multirow[t]{5}{*}{ DARK-EYED JUNCO (S-c) } & & 1 & & & & 3 & & & \\
\hline & & & & 100 & & 20 & 130 & & \\
\hline & & & 12 & 17 & & 29 & & & \\
\hline & & & & & & & & & \\
\hline & & & & & & & & & \\
\hline \multirow{2}{*}{$\begin{array}{l}\text { COMMON REDPOLL } \\
\text { HOARY REDPOLL }\end{array}$} & & & 12 & 6 & 2 & & 65 & & 8 \\
\hline & & & & & & & + & & \\
\hline \multirow{3}{*}{$\begin{array}{l}\text { PINE SISKIN } \\
\text { EVENING GROSBEAK } \\
\text { HOUSE SPARROW }\end{array}$} & & & & & & 1 & & & \\
\hline & & & & 17 & & & & & 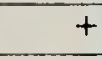 \\
\hline & 485 & 90 & 10 & & 33 & 86 & 13 & & 131 \\
\hline \multirow{5}{*}{$\begin{array}{l}\text { INDIVIDUALS IN TABLES } 4 \& 5 \\
\text { SPECIES IN TABLES } 4 \& 5 \\
\text { INDIVIDUALS ON COUNT DAY } \\
\text { SPECIES ON COUNT DAY } \\
\text { SPECIES COUNT PERIOD } \\
\end{array}$} & 0 & 1 & 0 & 1 & 35 & 8 & 0 & 128 & \\
\hline & 0 & 1 & 0 & 1 & 5 & 3 & 0 & 3 & 11 \\
\hline & 649 & 348 & 323 & 251 & 1176 & 768 & 322 & 3740 & 1663 \\
\hline & 15 & 14 & 13 & 14 & 20 & 19 & 13 & 14 & 28 \\
\hline & 16 & 15 & 14 & 18 & 20 & 19 & 14 & 14 & 39 \\
\hline
\end{tabular}


Table 3-4. SPECIES RECORDED FROM MORE THAN EIGHT LOCALITIES $(+=$ species seen during the count period but not on count day)

\begin{tabular}{|c|c|c|c|c|c|c|c|c|c|}
\hline 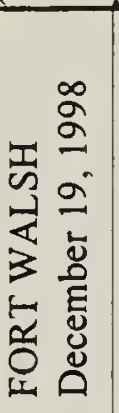 & 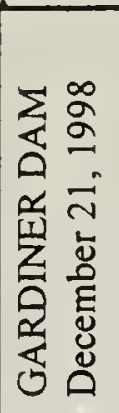 & 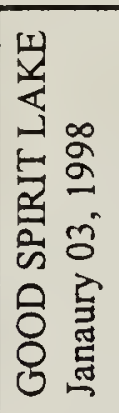 & 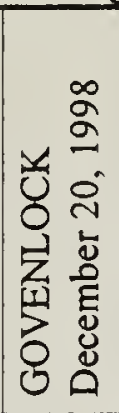 & 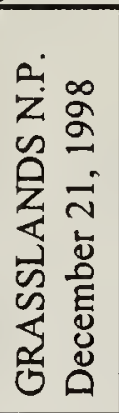 & 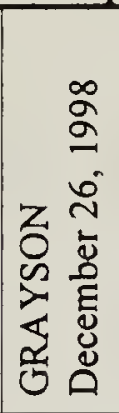 & 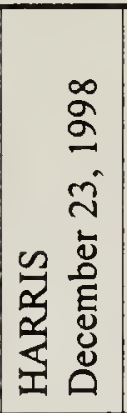 & 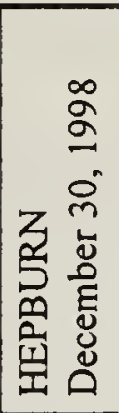 & 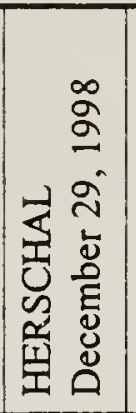 & 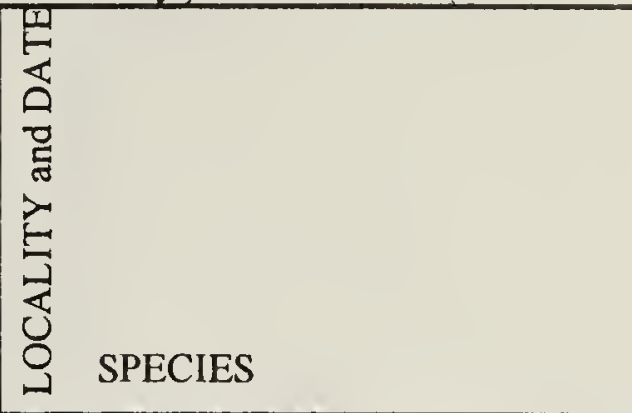 \\
\hline 2 & 251 & & & & & & & & CANADA GOOSE \\
\hline \multirow[t]{2}{*}{19} & 2219 & & & & 12 & & & & \multirow{4}{*}{$\begin{array}{l}\text { MALLARD } \\
\text { COMMON GOLDENEYE } \\
\text { BALD EAGLE } \\
\text { NORTHERN GOSHAWK }\end{array}$} \\
\hline & 53 & & & & 4 & & & & \\
\hline+ & 20 & & 2 & & 1 & 1 & & & \\
\hline 1 & & & & & & & & & \\
\hline 2 & 5 & & & & & & & 2 & GOLDEN EAGLE \\
\hline 1 & & & & & & & & & MERLIN \\
\hline 22 & 45 & + & 22 & & & 78 & & 6 & \multirow{3}{*}{$\begin{array}{l}\text { GRAY PARTRIDGE } \\
\text { RING-NECKED PHEASANT } \\
\text { RUFFED GROUSE }\end{array}$} \\
\hline 4 & & & 22 & 7 & & & & & \\
\hline 1 & & 2 & & & 1 & & & & \\
\hline \multirow[t]{2}{*}{5} & 37 & & 23 & 57 & & 3 & & & SHARP-TAILED GROUSE \\
\hline & 30 & 7 & & & 27 & 178 & & 84 & ROCK DOVE \\
\hline \multirow[t]{3}{*}{2} & 9 & 2 & 5 & 1 & & 1 & & 5 & GREAT HORNED OWL \\
\hline & 3 & & + & & & 1 & & 14 & \multirow{2}{*}{$\begin{array}{l}\text { SNOWY OWL } \\
\text { SHORT-EARED OWL }\end{array}$} \\
\hline & & & 7 & & & & & & \\
\hline 4 & & 4 & & & 3 & 2 & 1 & & \multirow{5}{*}{$\begin{array}{l}\text { DOWNY WOODPECKER } \\
\text { HAIRY WOODPECKER } \\
\text { BLACK-BACKED WOODPECKER } \\
\text { NORTHERN FLICKER (Y-s) } \\
\text { PILEATED WOODPECKER }\end{array}$} \\
\hline \multirow[t]{4}{*}{1} & 1 & 7 & & & 1 & & 1 & & \\
\hline & & & & & & & & & \\
\hline & & & & & & & & & \\
\hline & & & & & & & & & \\
\hline \multirow[t]{3}{*}{11} & 10 & & 276 & 302 & & 20 & & 23 & HORNED LARK \\
\hline & & & & & & & & & GRAY JAY \\
\hline & 6 & 2 & & & 2 & & 3 & & BLUE JAY \\
\hline 132 & 194 & 19 & 25 & 37 & 24 & 116 & 3 & 75 & BLACK-BILLED MAGPIE \\
\hline 1 & & 21 & & & 4 & 1 & + & & COMMON RAVEN \\
\hline \multirow[t]{2}{*}{52} & 7 & 37 & 1 & & 23 & 10 & 5 & 3 & BLACK-CAPPED CHICKADEE \\
\hline & & & & & & & & & BOREAL CHICKADEE \\
\hline \multirow[t]{2}{*}{1} & & & & & & & & & \multirow{3}{*}{$\begin{array}{l}\text { RED-BREASTED NUTHATCH } \\
\text { WHITE-BREASTED NUTHATCH } \\
\text { GOLDEN-CROWNED KINGLET }\end{array}$} \\
\hline & & 4 & & & 3 & & & & \\
\hline 2 & & & & & & 1 & & & \\
\hline & & & & & 1 & & & & AMERICAN ROBIN \\
\hline 27 & 27 & & 2 & & & 17 & & & BOHEMIAN WAXWING \\
\hline 1 & & & & 1 & & & & & NORTHERN SHRIKE \\
\hline 3 & & & 3 & & & & & & EUROPEAN STARLING \\
\hline & & & & & & & & & WHITE-THROATED SPARROW \\
\hline & & & & & & 5 & 1 & & DARK-EYED JUNCO (S-c) \\
\hline 114 & 273 & & 161 & & 71 & 32 & + & 1354 & SNOW BUNTING \\
\hline 23 & 3 & 14 & & & & & & & PINE GROSBEAK \\
\hline & & & & & & & & & HOUSE FINCH \\
\hline 1 & & & & & & & & & RED CROSSBILL \\
\hline 122 & 6 & & 15 & & & 3 & & 109 & COMMON REDPOLL \\
\hline 3 & & & & & & & & & HOARY REDPOLL \\
\hline & & & & & & & & & PINE SISKIN \\
\hline & 5 & & & & & & & & EVENING GROSBEAK \\
\hline 150 & 468 & 68 & 210 & & 15 & 875 & 12 & 149 & HOUSE SPARROW \\
\hline 70 & 245 & 0 & 24 & T & T & T & 0 & 0 & INDIVIDUALS IN TABLES $4 \& 5$ \\
\hline 14 & 9 & 0 & 4 & 1 & 1 & 1 & 0 & 0 & SPECIES IN TABLES 4 \& 5 \\
\hline 777 & 3917 & 187 & 798 & 406 & 193 & 1345 & 26 & 1824 & INDIVIDUALS ON COUNT DAY \\
\hline 38 & 28 & 12 & 18 & 7 & 16 & 18 & 7 & 11 & SPECIES ON COUNT DAY \\
\hline 40 & 28 & 13 & 19 & 7 & 16 & 18 & 9 & 11 & SPECIES COUNT PERIOD \\
\hline
\end{tabular}


Table 3-5. SPECIES RECORDED FROM MORE THAN EIGHT LOCALITIES $(+=$ species seen during the count period but not on count day)

SPECIES

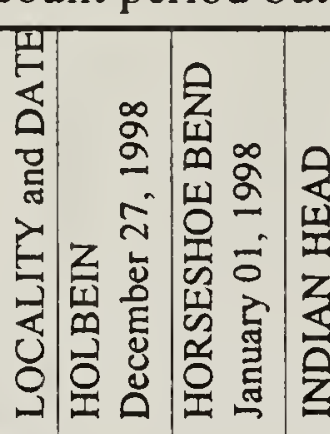

CANADA GOOSE

MALLARD

COMMON GOLDENEYE

BALD EAGLE

NORTHERN GOSHAWK

GOLDEN EAGLE

MERLIN

GRAY PARTRIDGE

RING-NECKED PHEASANT

RUFFED GROUSE

SHARP-TAILED GROUSE

ROCK DOVE

GREAT HORNED OWL

SNOWY OWL

SHORT-EARED OWL

DOWNY WOODPECKER

HAIRY WOODPECKER

BLACK-BACKED WOODPECKER

NORTHERN FLICKER (Y-s)

PILEATED WOODPECKER

HORNED LARK

GRAY JAY

BLUE JAY

BLACK-BILLED MAGPIE

COMMON RAVEN

BLACK-CAPPED CHICKADEE

BOREAL CHICKADEE

RED-BREASTED NUTHATCH

WHITE-BREASTED NUTHATCH

GOLDEN-CROWNED KINGLET

AMERICAN ROBIN

BOHEMIAN WAXWING

NORTHERN SHRIKE

EUROPEAN STARLING

WHITE-THROATED SPARROW

DARK-EYED JUNCO (S-c)

SNOW BUNTING

PINE GROSBEAK

HOUSE FINCH

RED CROSSBILL

COMMON REDPOLL

HOARY REDPOLL

PINE SISKIN

EVENING GROSBEAK

HOUSE SPARROW

INDIVIDUALS IN TABLES 4 \& 5

SPECIES IN TABLES 4 \& 5

INDIVIDUALS ON COUNT DAY

SPECIES ON COUNT DAY

SPECIES COUNT PERIOD

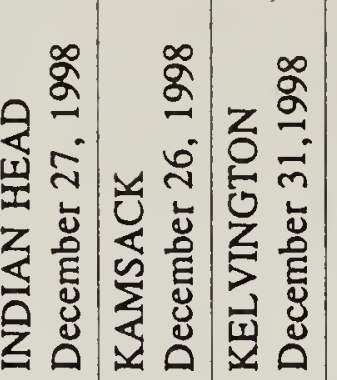

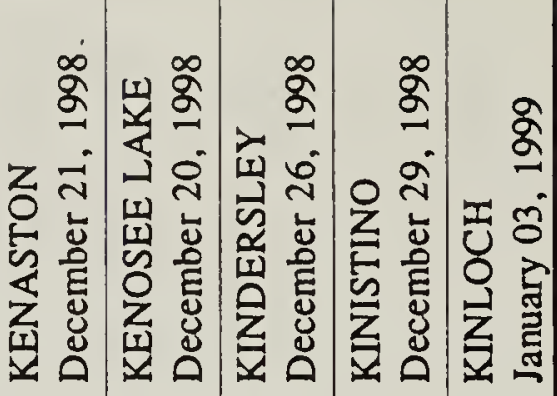

\begin{tabular}{|c|c|c|c|c|c|c|c|c|c|}
\hline & & 18 & & & & & & & \\
\hline & & 70 & & & & & & & \\
\hline & & 15 & & & & & & & \\
\hline & & 4 & 1 & & & & & & \\
\hline & & 1 & & & & & & & \\
\hline & & & & & + & & + & & \\
\hline & & 1 & & & & & & & \\
\hline & & 12 & & 8 & 14 & & 8 & & \\
\hline & & & & & & & & & \\
\hline & & & & + & & 1 & & & + \\
\hline & & 17 & & + & 18 & & 12 & & \\
\hline & & 49 & & 5 & 24 & & 17 & 21 & + \\
\hline & & 2 & & & & & 1 & & + \\
\hline & & & & & & & + & & \\
\hline & & & & & & & & & \\
\hline 2 & & 7 & 1 & 3 & 1 & 5 & & & 5 \\
\hline 3 & & 7 & 3 & 4 & + & 7 & & & 6 \\
\hline & & & & & & & & & \\
\hline & & & & & & & & & \\
\hline & & & 3 & & & & & & + \\
\hline & & & & & 3 & & 9 & & \\
\hline
\end{tabular}

\begin{tabular}{|r|r|r|r|r|r|r|r|r|r|r|}
\hline & 5 & & & & & & & & 6 \\
\hline 4 & 5 & 8 & 62 & 7 & 4 & 20 & & & 18 \\
\hline 1 & 8 & 72 & 6 & 14 & 12 & 27 & 25 & 4 & 9 \\
\hline 1 & 8 & 31 & 4 & 28 & 2 & 9 & & 2 & 18 \\
\hline 8 & 14 & 70 & 63 & 32 & 4 & 139 & & 3 & 36 \\
\hline 1 & 2 & & & & & & & & + \\
\hline
\end{tabular}

\begin{tabular}{|r|r|r|r|r|r|r|r|r|r|}
\hline 1 & 2 & & & & & & & & + \\
\hline & & 18 & 2 & & & & & & 2 \\
\hline 1 & & 8 & 4 & & & 8 & & & 7 \\
\hline & & 2 & & & & 1 & & & \\
\hline & & 2 & & & & & & & \\
\hline & & & 1 & & & & & & 1 \\
\hline
\end{tabular}

\begin{tabular}{|r|r|r|r|r|r|r|r|r|r|}
\hline & & & & & & & & & \\
\hline 2 & & & & & & & & & \\
\hline & 50 & 51 & 100 & 200 & 49 & & + & & 90 \\
\hline & 21 & & 43 & & & 2 & & & 31 \\
\hline & & & & & & & & & \\
\hline 12 & 23 & 2 & 45 & 80 & 32 & & & & 23 \\
\hline 10 & & 20 & & & & & & & \\
\hline & 4 & 20 & 10 & + & & 44 & & & \\
\hline 35 & & & 270 & & & 45 & & & 10 \\
\hline & & 80 & 100 & & 34 & 10 & 45 & 71 & 22 \\
\hline 0 & 1 & 4 & 2 & 0 & 1 & 19 & 0 & 4 & 0 \\
\hline 0 & 1 & 4 & 2 & 0 & 1 & 2 & 0 & 1 & 0 \\
\hline 80 & 141 & 2091 & 768 & 383 & 254 & 337 & 117 & 105 & 284 \\
\hline 12 & 11 & 30 & 21 & 11 & 14 & 15 & 7 & 6 & 15 \\
\hline 12 & 11 & 30 & 21 & 15 & 16 & 15 & 10 & 6 & 20 \\
\hline
\end{tabular}


Table 3-6. SPECIES RECORDED FROM MORE THAN EIGHT LOCALITIES ( $t=$ species seen during the count period but not on count day)

\begin{tabular}{|c|c|c|c|c|c|c|c|c|c|}
\hline 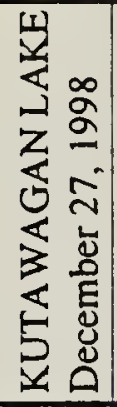 & 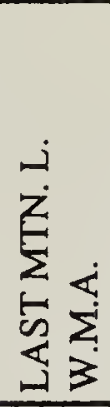 & 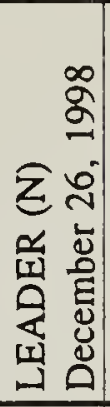 & 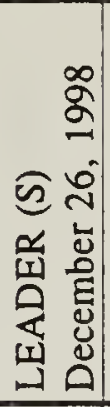 & 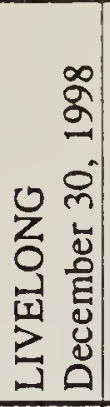 & 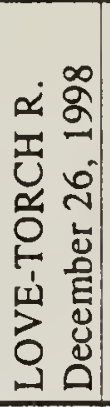 & 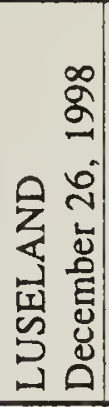 & 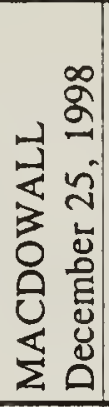 & 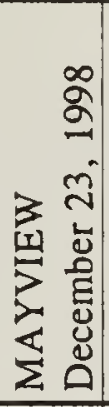 & 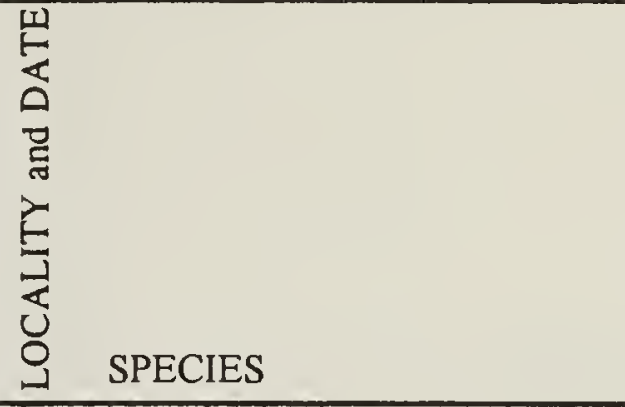 \\
\hline & & & & & & & & & \multirow{5}{*}{$\begin{array}{l}\text { CANADA GOOSE } \\
\text { MALLARD } \\
\text { COMMON GOLDENEYE } \\
\text { BALD EAGLE } \\
\text { NORTHERN GOSHAWK }\end{array}$} \\
\hline & & & & & & & & & \\
\hline & & & & & & & & & \\
\hline & & & 1 & & & & & & \\
\hline & & & & & 1 & & & & \\
\hline & & 1 & & & + & 1 & & & \multirow{5}{*}{$\begin{array}{l}\text { GOLDEN EAGLE } \\
\text { MERLIN } \\
\text { GRAY PARTRIDGE } \\
\text { RING-NECKED PHEASANT } \\
\text { RUFFED GROUSE }\end{array}$} \\
\hline & & & & & & & & & \\
\hline+ & 16 & 10 & 12 & & & 46 & & & \\
\hline & & 30 & 4 & & & & & & \\
\hline & & & & 5 & 8 & & 1 & & \\
\hline 25 & 4 & 2 & & & & & & & \multirow{5}{*}{$\begin{array}{l}\text { SHARP-TAILED GROUSE } \\
\text { ROCK DOVE } \\
\text { GREAT HORNED OWL } \\
\text { SNOWY OWL } \\
\text { SHORT-EARED OWL }\end{array}$} \\
\hline 4 & 256 & & 30 & 40 & + & 119 & 25 & & \\
\hline+ & 1 & & & & & 2 & & & \\
\hline 3 & 2 & & 2 & & & & & & \\
\hline & 3 & & 2 & & & 1. & & & \\
\hline & 1 & 1 & 1 & 2 & 8 & + & 2 & & \multirow{5}{*}{$\begin{array}{l}\text { DOWNY WOODPECKER } \\
\text { HAIRY WOODPECKER } \\
\text { BLACK-BACKED WOODPECKER } \\
\text { NORTHERN FLICKER (Y-s) } \\
\text { PILEATED WOODPECKER }\end{array}$} \\
\hline & & & 1 & 6 & 10 & + & 2 & & \\
\hline & & & & & & & & & \\
\hline & & & & & & + & & & \\
\hline & & & & & 2 & & & & \\
\hline & & & 3 & & & 6 & & & \multirow{5}{*}{$\begin{array}{l}\text { HORNED LARK } \\
\text { GRAY JAY } \\
\text { BLUE JAY } \\
\text { BLACK-BILLED }\end{array}$} \\
\hline & & & & & 14 & & & & \\
\hline & & & 2 & 8 & 28 & 3 & 4 & 2 & \\
\hline 95 & 63 & 17 & 85 & & 30 & 53 & 4 & 10 & \\
\hline & & & & 25 & 83 & + & 6 & 2 & \\
\hline 10 & & & 4 & 20 & 56 & 4 & 25 & 6 & \multirow{5}{*}{$\begin{array}{l}\text { BLACK-CAPPED CHICKADEE } \\
\text { BOREAL CHICKADEE } \\
\text { RED-BREASTED NUTHATCH } \\
\text { WHITE-BREASTED NUTHATCH } \\
\text { GOLDEN-CROWNED KINGLET }\end{array}$} \\
\hline & & & & & & & & 1 & \\
\hline & & & & & 3 & + & & 1 & \\
\hline & & & & & 9 & & & 3 & \\
\hline & & & & & & + & & & \\
\hline & & & & & & & & & \multirow{5}{*}{$\begin{array}{l}\text { AMERICAN ROBIN } \\
\text { BOHEMIAN WAXWING } \\
\text { NORTHERN SHRIKE } \\
\text { EUROPEAN STARLING } \\
\text { WHITE-THROATED SPARROW }\end{array}$} \\
\hline 26 & 65 & & & + & & 17 & & & \\
\hline & & & & & & & & & \\
\hline & & & 1 & & & & & & \\
\hline & & & & & & & & & \\
\hline & & & & & + & & & + & \multirow{5}{*}{$\begin{array}{l}\text { DARK-EYED JUNC } \\
\text { SNOW BUNTING } \\
\text { PINE GROSBEAK } \\
\text { HOUSE FINCH } \\
\text { RED CROSSBILL }\end{array}$} \\
\hline 31 & 5541 & + & & & 340 & 355 & & & \\
\hline & & & & 35 & 48 & & 15 & 3 & \\
\hline & & & & & & & & & \\
\hline & & & & & & 5 & & & \\
\hline 20 & + & 12 & & 30 & 65 & 137 & 2 & & \multirow{5}{*}{$\begin{array}{l}\text { COMMON REDPOLL } \\
\text { HOARY REDPOLL } \\
\text { PINE SISKIN } \\
\text { EVENING GROSBEAK } \\
\text { HOUSE SPARROW }\end{array}$} \\
\hline & & & & & & & & & \\
\hline & & & & & & & & & \\
\hline & & & & 10 & 10 & & 2 & & \\
\hline 567 & 446 & 8 & 216 & 20 & 151 & 394 & & & \\
\hline 1 & $T$ & 0 & 6 & 0 & 7 & 2 & 2 & 2 & \multirow{5}{*}{$\begin{array}{l}\text { INDIVIDUALS IN TABLES } 4 \& 5 \\
\text { SPECIES IN TABLES } 4 \text { \& } 5 \\
\text { INDIVIDUALS ON COUNT DAY } \\
\text { SPECIES ON COUNT DAY } \\
\text { SPECIES COUNT PERIOD }\end{array}$} \\
\hline 1 & 1 & 2 & 3 & 0 & 3 & 2 & 1 & 1 & \\
\hline 782 & 6399 & 81 & 370 & 201 & 873 & 1145 & 90 & 30 & \\
\hline 10 & 12 & 8 & 17 & 11 & 19 & 16 & 12 & 9 & \\
\hline 12 & 13 & 11 & 17 & 12 & 23 & 22 & 12 & 10 & \\
\hline
\end{tabular}


Table 3-7. SPECIES RECORDED FROM MORE THAN EIGHT LOCALITIES

$(+=$ species seen during the count period but not on count day)

\begin{tabular}{|c|c|c|c|c|c|c|c|c|c|}
\hline 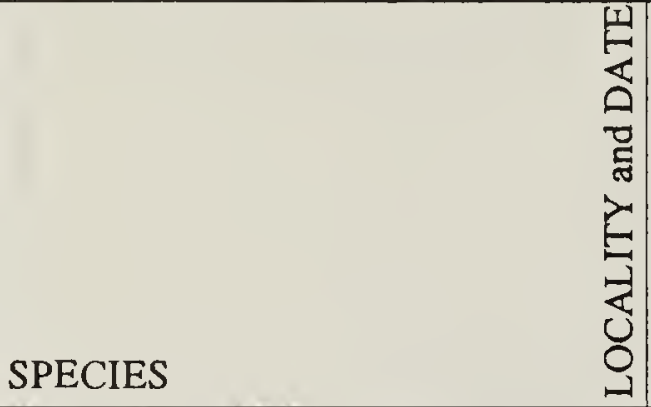 & 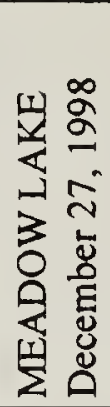 & 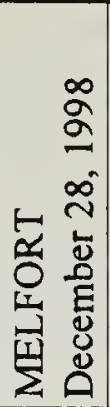 & 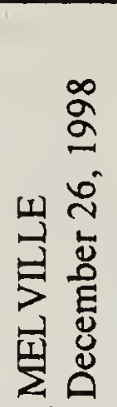 & 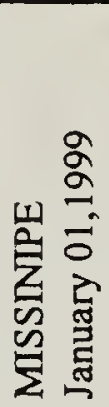 & 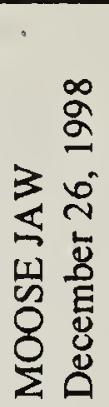 & 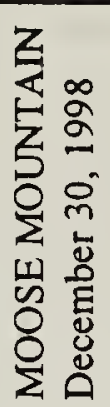 & 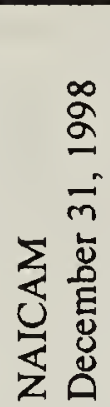 & 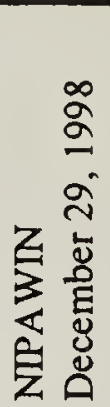 & 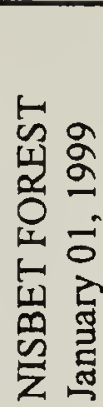 \\
\hline \multicolumn{10}{|l|}{ CANADA GOOSE } \\
\hline \\
\hline \multicolumn{10}{|l|}{ COMMON GOLDENEYE } \\
\hline \multirow{2}{*}{\multicolumn{10}{|c|}{$\begin{array}{l}\text { BALD EAGLE } \\
\text { NORTHERN GOSHAWK }\end{array}$}} \\
\hline & & & & & & & & & \\
\hline \multicolumn{10}{|l|}{ GOLDEN EAGLE } \\
\hline \multirow{4}{*}{$\begin{array}{l}\text { MERLIN } \\
\text { GRAY PARTRIDGE } \\
\text { RING-NECKED PHEASANT } \\
\text { RUFFED GROUSE }\end{array}$} & & & & & 1 & & & & \\
\hline & & & & & 8 & & & & \\
\hline & & & & & 1 & & & & \\
\hline & & & & & & & & & 3 \\
\hline \multirow{5}{*}{$\begin{array}{l}\text { SHARP-TAILED GROUSE } \\
\text { ROCK DOVE } \\
\text { GREAT HORNED OWL } \\
\text { SNOWY OWL } \\
\text { SHORT-EARED OWL }\end{array}$} & 22 & & & & & & & & \\
\hline & 160 & & 30 & & 150 & 16 & & & \\
\hline & & 1 & 1 & & 1 & & & & + \\
\hline & & & & & 1 & & & & \\
\hline & & & & & & & & & \\
\hline \multirow{5}{*}{$\begin{array}{l}\text { DOWNY WOODPECKER } \\
\text { HAIRY WOODPECKER } \\
\text { BLACK-BACKED WOODPECKER } \\
\text { NORTHERN FLICKER (Y-s) } \\
\text { PILEATED WOODPECKER }\end{array}$} & 3 & 1 & 2 & & 4 & 10 & 2 & 2 & 1 \\
\hline & 2 & & 2 & 1 & 4 & 5 & 2 & 2 & 2 \\
\hline & & & & & & & & & \\
\hline & & & & & 2 & & & & \\
\hline & & & & & & & & & + \\
\hline \multirow{5}{*}{$\begin{array}{l}\text { HORNED LARK } \\
\text { GRAY JAY } \\
\text { BLUE JAY } \\
\text { BLACK-BILLED MAGPIE } \\
\text { COMMON RAVEN }\end{array}$} & & & & & 40 & & & & \\
\hline & 3 & & & 2 & & & & & 2 \\
\hline & 6 & 3 & 1 & 3 & & 35 & 2 & 4 & 14 \\
\hline & 17 & 5 & 4 & & 47 & 112 & 4 & 7 & 24 \\
\hline & 65 & 4 & 2 & & & 10 & 2 & 126 & 13 \\
\hline \multirow{5}{*}{$\begin{array}{l}\text { BLACK-CAPPED CHICKADEE } \\
\text { BOREAL CHICKADEE } \\
\text { RED-BREASTED NUTHATCH } \\
\text { WHITE-BREASTED NUTHATCH } \\
\text { GOLDEN-CROWNED KINGLET }\end{array}$} & 34 & 2 & 18 & 2 & 35 & 130 & 6 & 6 & 12 \\
\hline & & & & 1 & & & & 2 & \\
\hline & 1 & & & & 24 & & & 2 & \\
\hline & 1 & & & & 2 & 1 & & & 2 \\
\hline & & & & & & & & & \\
\hline \multirow{5}{*}{$\begin{array}{l}\text { AMERICAN ROBIN } \\
\text { BOHEMIAN WAXWING } \\
\text { NORTHERN SHRIKE } \\
\text { EUROPEAN STARLING } \\
\text { WHITE-THROATED SPARROW }\end{array}$} & & & & & 2 & & & & \\
\hline & 7 & & & & 45 & & & 8 & \\
\hline & & & & & & & & & \\
\hline & & & & & & 8 & & & \\
\hline & & & & & 2 & & & & \\
\hline \multirow{5}{*}{$\begin{array}{l}\text { DARK-EYED JUNCO }(\mathrm{S}-\mathrm{c}) \\
\text { SNOW BUNTING } \\
\text { PINE GROSBEAK } \\
\text { HOUSE FINCH } \\
\text { RED CROSSBILL }\end{array}$} & & & & & 7 & & & & \\
\hline & 55 & & & & & 1 & & 55 & 530 \\
\hline & 64 & & & & 1 & & & 2 & 38 \\
\hline & & & & & 92 & & & & \\
\hline & 4 & & & & & & & & \\
\hline \multirow{5}{*}{$\begin{array}{l}\text { COMMON REDPOLL } \\
\text { HOARY REDPOLL } \\
\text { PINE SISKIN } \\
\text { EVENING GROSBEAK } \\
\text { HOUSE SPARROW }\end{array}$} & 100 & & & & & & & + & 9 \\
\hline & 4 & & & & & & & & 1 \\
\hline & & & 3 & & 30 & 27 & & & \\
\hline & 33 & & & + & & 10 & & 20 & 22 \\
\hline & 277 & 150 & 60 & & 362 & 94 & 15 & & 47 \\
\hline \multirow{5}{*}{$\begin{array}{l}\text { INDIVIDUALS IN TABLES } 4 \& 5 \\
\text { SPECIES IN TABLES } 4 \text { \& } 5 \\
\text { INDIVIDUALS ON COUNT DAY } \\
\text { SPECIES ON COUNT DAY } \\
\text { SPECIES COUNT PERIOD }\end{array}$} & 0 & 0 & 0 & 0 & 1 & 4 & 0 & 0 & 0 \\
\hline & 0 & 0 & 0 & 0 & 1 & 2 & 0 & 1 & 0 \\
\hline & 858 & 166 & 123 & 9 & 862 & 463 & 33 & 236 & 720 \\
\hline & 19 & 7 & 10 & 5 & 23 & 15 & 7 & 12 & 15 \\
\hline & 19 & 7 & 10 & 6 & 23 & 15 & 7 & 15 & 17 \\
\hline
\end{tabular}


Table 3-8. SPECIES RECORDED FROM MORE THAN EIGHT LOCALITIES

$(+=$ species seen during the count period but not on count day)

\begin{tabular}{|c|c|c|c|c|c|c|c|c|c|}
\hline 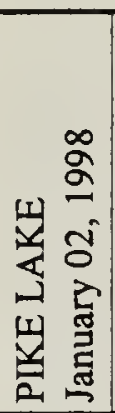 & 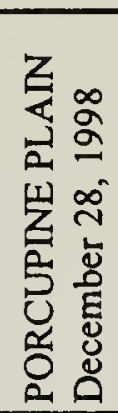 & 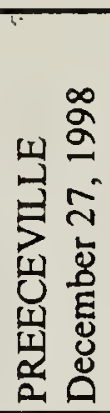 & 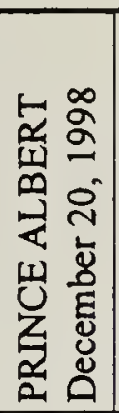 & 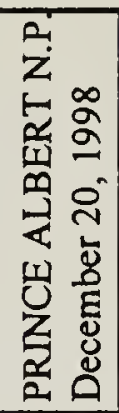 & 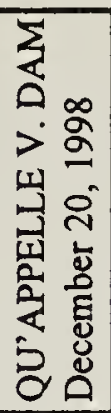 & 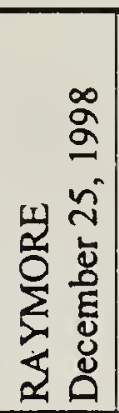 & 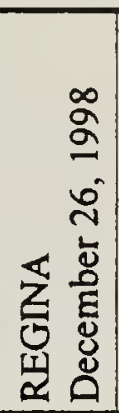 & 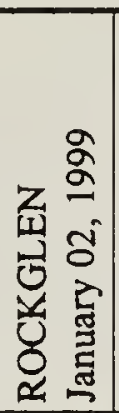 & 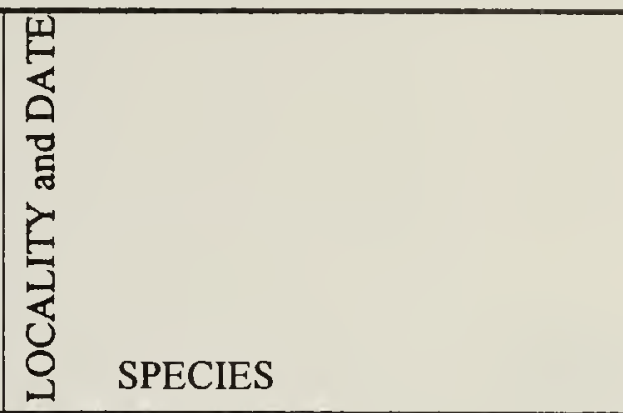 \\
\hline & & & & & 905 & & + & & CANADA GOOSE \\
\hline & & & & & 380 & 1 & 6 & & MALLARD \\
\hline & & & & & 24 & & & & COMMON GOLDENEYE \\
\hline & & & & & 2 & & & & BALD EAGLE \\
\hline 1 & & 1 & 1 & & & & & & NORTHERN GOSHAWK \\
\hline & & & & & 2 & & & 1 & GOLDEN EAGLE \\
\hline & & & 2 & & & 1 & 1 & & MERLIN \\
\hline 3 & & & & & & 17 & 121 & 42 & GRAY PARTRIDGE \\
\hline & & & & & & & 3 & & RING-NECKED PHEASANT \\
\hline 1 & & 2 & 8 & 12 & & 2 & & & RUFFED GROUSE \\
\hline 39 & & & 13 & & 29 & 7 & 34 & 49 & SHARP-TAILED GROUSE \\
\hline 39 & & 23 & 286 & & 22 & 11 & 563 & & ROCK DOVE \\
\hline 2 & & & & & 2 & 1 & 9 & & GREAT HORNED OWL \\
\hline & & & & & & + & 8 & & SNOWY OWL \\
\hline & & & & & & & & + & SHORT-EARED OWL \\
\hline 21 & & 9 & 19 & & & 2 & 22 & & DOWNY WOODPECKER \\
\hline 39 & 1 & 7 & 18 & 3 & & 2 & 5 & & HAIRY WOODPECKER \\
\hline & & & & 2 & & & & & BLACK-BACKED WOODPECKER \\
\hline 3 & & & & & & & 2 & & NORTHERN FLICKER (Y-s) \\
\hline 1 & 1 & & 2 & 2 & & & & & PILEATED WOODPECKER \\
\hline 1 & & & & & & 1 & & 268 & HORNED LARK \\
\hline & & 7 & 15 & 7 & & & & & GRAY JAY \\
\hline 40 & & 8 & 28 & 1 & 4 & & 6 & & BLUE JAY \\
\hline 217 & 2 & 41 & 133 & 1 & 48 & 77 & 258 & 11 & BLACK-BILLED MAGPIE \\
\hline 40 & 4 & 52 & 226 & 11 & 1 & 17 & & & COMMON RAVEN \\
\hline 357 & 9 & 46 & 119 & 14 & 3 & 21 & 40 & + & BLACK-CAPPED CHICKADEE \\
\hline & & 5 & 4 & 28 & & & & & BOREAL CHICKADEE \\
\hline 2 & & 2 & 4 & & & & 31 & & RED-BREASTED NUTHATCH \\
\hline 7 & 1 & 6 & 11 & & & & 9 & & WHITE-BREASTED NUTHATCH \\
\hline & & & & & & & 5 & & GOLDEN-CROWNED KINGLET \\
\hline 3 & & & & & & & 3 & & AMERICAN ROBIN \\
\hline 161 & & & 256 & & 24 & 7 & 1133 & 6 & BOHEMIAN WAXWING \\
\hline 1 & & 2 & & & & + & & & NORTHERN SHRIKE \\
\hline & & & & & & 21 & 108 & & EUROPEAN STARLING \\
\hline & & & 1 & & & & 1 & & WHITE-THROATED SPARROW \\
\hline 6 & & & & & & 2 & 15 & & DARK-EYED JUNCO (S-c) \\
\hline 199 & & 143 & 71 & & & 2 & 625 & & SNOW BUNTING \\
\hline 6 & 5 & 26 & 79 & & & 2 & & & PINE GROSBEAK \\
\hline & & & & & & & 190 & & HOUSE FINCH \\
\hline & & & & 2 & & & + & & RED CROSSBILL \\
\hline 94 & 3 & 34 & 159 & 5 & & 3 & & & COMMON REDPOLL \\
\hline & & & & & & & & & HOARY REDPOLL \\
\hline 2 & & & & & & & 95 & & PINE SISKIN \\
\hline 3 & 10 & 45 & 48 & & & 2 & & & EVENING GROSBEAK \\
\hline 353 & 15 & 42 & 316 & & 315 & 430 & 1097 & & HOUSE SPARROW \\
\hline 10 & 0 & 1 & 0 & 2 & 25 & 0 & 45 & 6 & INDIVIDUALS IN TABLES $4 \& 5$ \\
\hline 4 & 0 & 1 & 1 & 1 & 2 & 1 & 10 & 4 & SPECIES IN TABLES 4 \& 5 \\
\hline 1651 & 51 & 502 & 1819 & 90 & 1786 & 629 & 4435 & 383 & INDIVIDUALS ON COUNT DAY \\
\hline 31 & 10 & 20 & 23 & 13 & 16 & 21 & 33 & 8 & SPECIES ON COUNT DAY \\
\hline 31 & 10 & 20 & 24 & 13 & 16 & 24 & 37 & 12 & SPECIES COUNT PERIOD \\
\hline
\end{tabular}


Table 3-9. SPECIES RECORDED FROM MORE THAN EIGHT LOCALITIES

$(+=$ species seen during the count period but not on count day)

\begin{tabular}{|c|c|c|c|c|c|c|c|c|c|}
\hline 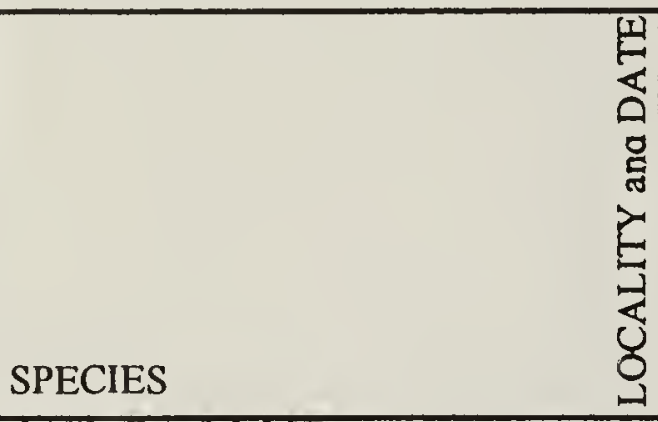 & 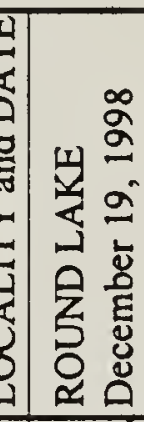 & 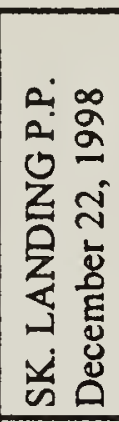 & 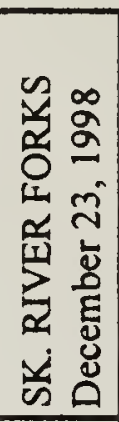 & 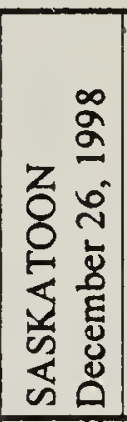 & 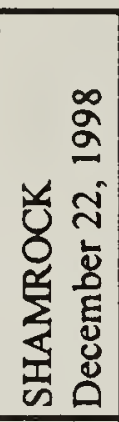 & 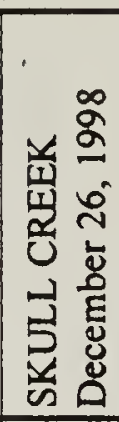 & 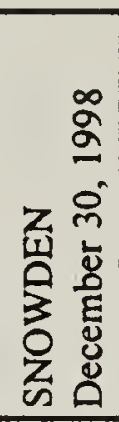 & 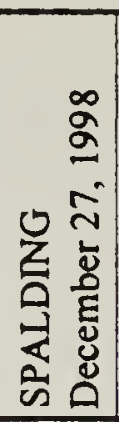 & 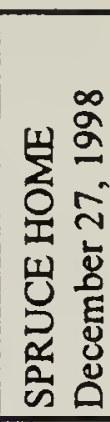 \\
\hline \multirow{5}{*}{$\begin{array}{l}\text { CANADA GOOSE } \\
\text { MALLARD } \\
\text { COMMON GOLDENEYE } \\
\text { BALD EAGLE } \\
\text { NORTHERN GOSHAWK }\end{array}$} & 2 & & & 63 & & & & & \\
\hline & 83 & 7 & & 59 & & & & & \\
\hline & 10 & & & 71 & & & & & \\
\hline & 1 & + & & + & & 2 & & & \\
\hline & & & & & & & & & \\
\hline \multirow{5}{*}{$\begin{array}{l}\text { GOLDEN EAGLE } \\
\text { MERLIN } \\
\text { GRAY PARTRIDGE } \\
\text { RING-NECKED PHEASANT } \\
\text { RUFFED GROUSE }\end{array}$} & 3 & 1 & & & & & & & \\
\hline & & & & 4 & & & & & \\
\hline & & 18 & & 39 & 9 & & 15 & & \\
\hline & & & & & & 7 & & & \\
\hline & 1 & & 1 & 1 & & & 24 & 1 & \\
\hline \multirow{5}{*}{$\begin{array}{l}\text { SHARP-TAILED GROUSE } \\
\text { ROCK DOVE } \\
\text { GREAT HORNED OWL } \\
\text { SNOWY OWL } \\
\text { SHORT-EARED OWL }\end{array}$} & 1 & 26 & & 30 & & 17 & & & \\
\hline & 3 & 2 & & 2090 & & 3 & 15 & 6 & \\
\hline & 1 & 2 & & 2 & & 1 & & & \\
\hline & & & & 1 & & & & & \\
\hline & & & & & & & & & \\
\hline \multirow{5}{*}{$\begin{array}{l}\text { DOWNY WOODPECKER } \\
\text { HAIRY WOODPECKER } \\
\text { BLACK-BACKED WOODPECKER } \\
\text { NORTHERN FLICKER (Y-s) } \\
\text { PILEATED WOODPECKER }\end{array}$} & 7 & 1 & 2 & 35 & & 5 & 8 & & 2 \\
\hline & 11 & & 2 & 33 & & 3 & 11 & 1 & 2 \\
\hline & & & & & & & 1 & & \\
\hline & & & & 20 & & 2 & & & \\
\hline & & & & & & & 1 & & \\
\hline \multirow{5}{*}{$\begin{array}{l}\text { HORNED LARK } \\
\text { GRAY JAY } \\
\text { BLUE JAY } \\
\text { BLACK-BILLED MAGPIE } \\
\text { COMMON RAVEN }\end{array}$} & & 346 & & & 216 & 99 & & & \\
\hline & & & & 2 & & & 13 & & \\
\hline & 9 & & 2 & 111 & & 3 & 28 & + & \\
\hline & 60 & 66 & 27 & 976 & 3 & 88 & 37 & 4 & \\
\hline & 12 & & 12 & 41 & & & 172 & 6 & \\
\hline \multirow{5}{*}{$\begin{array}{l}\text { BLACK-CAPPED CHICKADEE } \\
\text { BOREAL CHICKADEE } \\
\text { RED-BREASTED NUTHATCH } \\
\text { WHITE-BREASTED NUTHATCH } \\
\text { GOLDEN-CROWNED KINGLET }\end{array}$} & 138 & 15 & 23 & 640 & & 38 & 123 & 4 & 12 \\
\hline & & & 7 & & & & 13 & & \\
\hline & 1 & & & 74 & & & 1 & & \\
\hline & 8 & & 1 & 10 & & & 1 & & \\
\hline & 2 & & & 6 & & & & & \\
\hline \multirow{5}{*}{$\begin{array}{l}\text { AMERICAN ROBIN } \\
\text { BOHEMIAN WAXWING } \\
\text { NORTHERN SHRIKE } \\
\text { EUROPEAN STARLING } \\
\text { WHITE-THROATED SPARROW }\end{array}$} & & & & 8 & & & & & \\
\hline & 35 & & & 9974 & & 70 & & & \\
\hline & & & & 3 & & & & & \\
\hline & & & & 40 & & & & & \\
\hline & & & & 2 & & & & & \\
\hline \multirow{5}{*}{$\begin{array}{l}\text { DARK-EYED JUNCO (S-c) } \\
\text { SNOW BUNTING } \\
\text { PINE GROSBEAK } \\
\text { HOUSE FINCH } \\
\text { RED CROSSBILL }\end{array}$} & & & & 41 & & 1 & & & \\
\hline & & 505 & 220 & 137 & & 45 & 3 & & 1 \\
\hline & & & 10 & 14 & & & 103 & & 5 \\
\hline & & & & 76 & & & & & \\
\hline & & & & 67 & & & & & \\
\hline \multirow{5}{*}{$\begin{array}{l}\text { COMMON REDPOLL } \\
\text { HOARY REDPOLL } \\
\text { PINE SISKIN } \\
\text { EVENING GROSBEAK } \\
\text { HOUSE SPARROW }\end{array}$} & 275 & 23 & 30 & 199 & & 26 & 210 & & 18 \\
\hline & & & & 1 & & & & & \\
\hline & & & & 78 & & & 9 & & \\
\hline & 2 & & & & & & 63 & & \\
\hline & 52 & 264 & 33 & 3567 & 62 & 89 & 50 & 40 & \\
\hline \multirow{5}{*}{$\begin{array}{l}\text { INDIVIDUALS IN TABLES } 4 \& 5 \\
\text { SPECIES IN TABLES } 4 \& 5 \\
\text { INDIVIDUALS ON COUNT DAY } \\
\text { SPECIES ON COUNT DAY } \\
\text { SPECIES COUNT PERIOD }\end{array}$} & 4 & 253 & 0 & 83 & 0 & 23 & 0 & 0 & \\
\hline & 2 & 4 & 0 & 10 & 0 & 3 & 1 & 0 & 0 \\
\hline & 721 & 1529 & 370 & 18598 & 290 & 522 & 901 & 62 & 42 \\
\hline & 24 & 16 & 13 & 44 & 4 & 20 & 21 & 7 & 8 \\
\hline & 24 & 18 & 13 & 45 & 4 & 20 & 22 & 8 & \\
\hline
\end{tabular}


Table 3-10. SPECIES RECORDED FROM MORE THAN EIGHT LOCALITIES

$(+=$ species seen during the count period but not on count day)

\begin{tabular}{|c|c|c|c|c|c|c|c|c|c|}
\hline 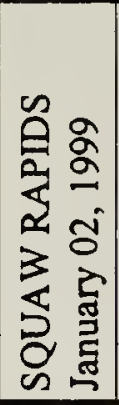 & 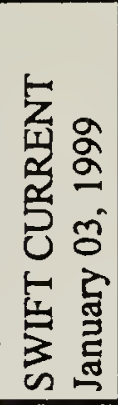 & 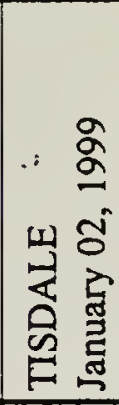 & 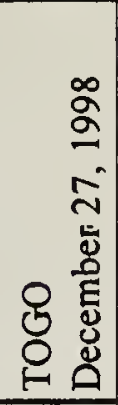 & 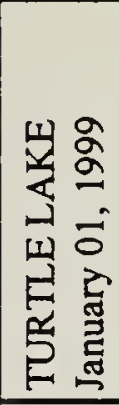 & 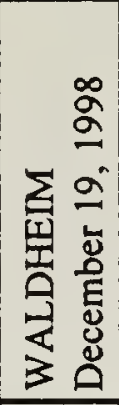 & 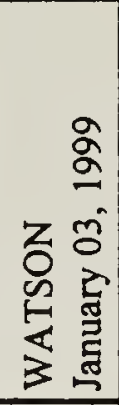 & 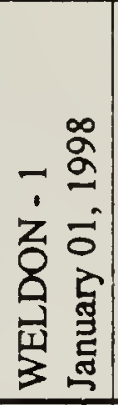 & 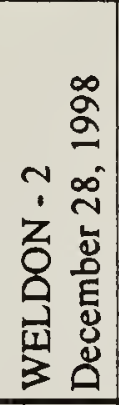 & 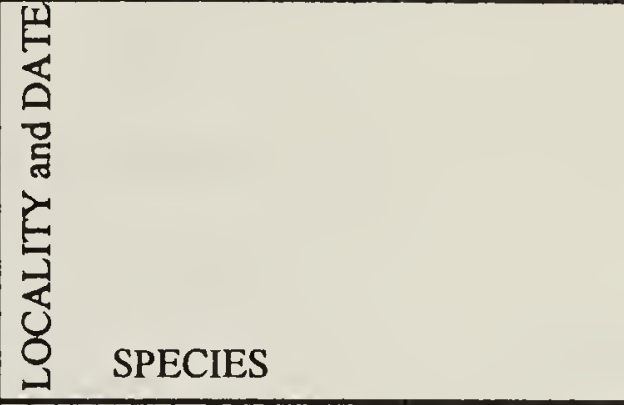 \\
\hline & & & & & & & & & \multirow{5}{*}{$\begin{array}{l}\text { CANADA GOOSE } \\
\text { MALLARD } \\
\text { COMMON GOLDENEYE } \\
\text { BALD EAGLE } \\
\text { NORTHERN GOSHAWK } \\
\end{array}$} \\
\hline 2 & 2 & & & & & & & & \\
\hline 255 & & & & & & & & & \\
\hline 5 & & & & & & & 1 & & \\
\hline 2 & & & & & & & & & \\
\hline & & & & 1 & & & & & \multirow{5}{*}{$\begin{array}{l}\text { GOLDEN EAGLE } \\
\text { MERLIN } \\
\text { GRAY PARTRIDGE } \\
\text { RING-NECKED PHEASANT } \\
\text { RUFFED GROUSE }\end{array}$} \\
\hline & & & & & & & & & \\
\hline & 56 & 7 & & & & & & & \\
\hline & 7 & & & & & & & & \\
\hline 7 & & & 3 & 10 & & & 1 & & \\
\hline 31 & 31 & & & 3 & 18 & 8 & & & \multirow{5}{*}{$\begin{array}{l}\text { SHARP-TAILED GROUSE } \\
\text { ROCK DOVE } \\
\text { GREAT HORNED OWL } \\
\text { SNOWY OWL } \\
\text { SHORT-EARED OWL }\end{array}$} \\
\hline & 317 & & & & 11 & & & & \\
\hline 1 & 1 & & & 1 & & & & 1 & \\
\hline & + & & & 1 & + & & & & \\
\hline & & & & & & & & & \\
\hline 2 & 5 & 3 & 2 & 3 & 1 & & 2 & & \multirow{5}{*}{$\begin{array}{l}\text { DOWNY WOODPECKER } \\
\text { HAIRY WOODPECKER } \\
\text { BLACK-BACKED WOODPECKER } \\
\text { NORTHERN FLICKER (Y-s) } \\
\text { PILEATED WOODPECKER }\end{array}$} \\
\hline 3 & & 3 & 3 & 4 & 2 & & 2 & & \\
\hline 1 & & & & & & & & & \\
\hline & 1 & & & & & & & & \\
\hline 4 & & & & 1 & & & & & \\
\hline & 444 & & & 4 & & & & & HORNED LARK \\
\hline 15 & & 1 & & 2 & & & & + & GRAY JAY \\
\hline 9 & & 5 & & 10 & 6 & & 4 & 1 & BLUE JAY \\
\hline 35 & 108 & 10 & 1 & 7 & 40 & 4 & 1 & 6 & BLACK-BILLED MAGPIE \\
\hline 46 & & 5 & 3 & 30 & 13 & 2 & 3 & 3 & COMMON RAVEN \\
\hline 23 & 5 & 25 & 8 & 7 & 12 & 10 & 8 & 30 & \multirow{5}{*}{$\begin{array}{l}\text { BLACK-CAPPED CHICKADEE } \\
\text { BOREAL CHICKADEE } \\
\text { RED-BREASTED NUTHATCH } \\
\text { WHITE-BREASTED NUTHATCH } \\
\text { GOLDEN-CROWNED KINGLET }\end{array}$} \\
\hline 7 & & & & 6 & & & & & \\
\hline & 6 & & & 1 & & & & & \\
\hline & + & & 1 & 3 & & & 2 & & \\
\hline & & & & & & & & & \\
\hline & 1 & & & & + & & & & \multirow{5}{*}{$\begin{array}{l}\text { AMERICAN ROBIN } \\
\text { BOHEMIAN WAXWING } \\
\text { NORTHERN SHRIKE } \\
\text { EUROPEAN STARLING } \\
\text { WHITE-THROATED SPARROW }\end{array}$} \\
\hline & 438 & & & & t & & & & \\
\hline & & & & & & & & & \\
\hline & & & & & & & & & \\
\hline & & & & & & & & & \\
\hline & 1 & & & 2 & & & & & \multirow{5}{*}{$\begin{array}{l}\text { DARK-EYED JUNCO }(\mathrm{S}-\mathrm{c}) \\
\text { SNOW BUNTING } \\
\text { PINE GROSBEAK } \\
\text { HOUSE FINCH } \\
\text { RED CROSSBILL }\end{array}$} \\
\hline 70 & 340 & 16 & 12 & 3 & & 40 & & & \\
\hline & & 9 & & 30 & & + & 10 & & \\
\hline & 10 & & & & & & & & \\
\hline & & & & & 8 & & & & \\
\hline 30 & & 27 & & & & & 30 & & \multirow{5}{*}{$\begin{array}{l}\text { COMMON REDPOLL } \\
\text { HOARY REDPOLL } \\
\text { PINE SISKIN } \\
\text { EVENING GROSBEAK } \\
\text { HOUSE SPARROW }\end{array}$} \\
\hline 4 & & 2 & & & & & & & \\
\hline & 22 & & & & + & & & & \\
\hline & & 7 & & 5 & & & 12 & & \\
\hline 15 & 1995 & & & 25 & 60 & 20 & & 35 & \\
\hline 11 & 37 & 7 & 0 & 4 & $T$ & 0 & 0 & 1 & INDIVIDUALS IN TABLES 4 \& 5 \\
\hline 4 & 2 & 1 & 0 & 2 & 1 & 0 & 0 & 1 & \multirow{4}{*}{$\begin{array}{l}\text { SPECIES IN TABLES } 4 \& 5 \\
\text { INDIVIDUALS ON COUNT DAY } \\
\text { SPECIES ON COUNT DAY } \\
\text { SPECIES COUNT PERIOD }\end{array}$} \\
\hline 578 & 3827 & 127 & 33 & 163 & 172 & 84 & 76 & 77 & \\
\hline 25 & 21 & 14 & 8 & 24 & 11 & 6 & 12 & 7 & \\
\hline 25 & 23 & 14 & 8 & 24 & 15 & 7 & 12 & 8 & \\
\hline
\end{tabular}


Table 3-11. SPECIES RECORDED FROM MORE THAN EIGHT LOCALITIES $(+=$ species seen during the count period but not on count day)

SPECIES

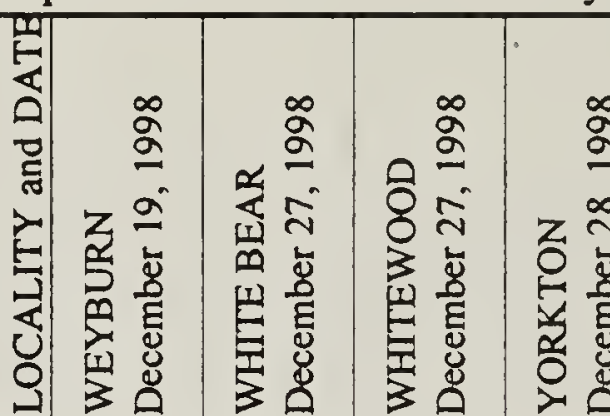

CANADA GOOSE

MALLARD

COMMON GOLDENEYE

BALD EAGLE

NORTHERN GOSHAWK

GOLDEN EAGLE

MERLIN

GRAY PARTRIDGE

RING-NECKED PHEASANT

RUFFED GROUSE

SHARP-TAILED GROUSE

ROCK DOVE

GREAT HORNED OWL

SNOWY OWL

SHORT-EARED OWL

DOWNY WOODPECKER

HAIRY WOODPECKER

BLACK-BACKED WOODPECKER

NORTHERN FLICKER (Y-s)

PILEATED WOODPECKER

HORNED LARK

GRAY JAY

BLUE JAY

BLACK-BILLED MAGPIE

COMMON RAVEN

BLACK-CAPPED CHICKADEE

BOREAL CHICKADEE

RED-BREASTED NUTHATCH

WHITE-BREASTED NUTHATCH

GOLDEN-CROWNED KINGLET

AMERICAN ROBIN

BOHEMIAN WAXWING

NORTHERN SHRIKE

EUROPEAN STARLING

WHITE-THROATED SPARROW

DARK-EYED JUNCO (S-c)

SNOW BUNTING

PINE GROSBEAK

HOUSE FINCH

RED CROSSBILL

COMMON REDPOLL

HOARY REDPOLL

PINE SISKIN

EVENING GROSBEAK

HOUSE SPARROW

INDIVIDUALS IN TABLES 4 \& 3

SPECIES IN TABLES $4 \& 5$

TOTAL INDIVIDUALS

TOTAL SPECIES ON COUNT DAY

TOTAL SPECIES COUNT PERIOD

\section{政}

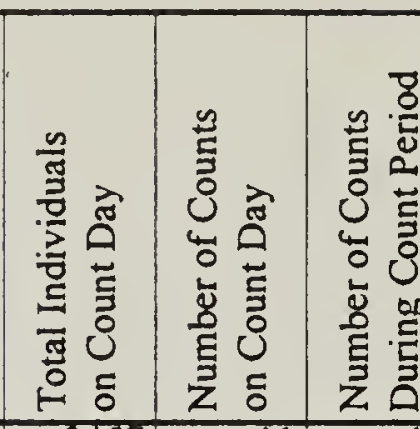

\begin{tabular}{|r|r|r|r|r|r|}
\hline & & & 3457 & 11 & 12 \\
\hline & & 20 & 18836 & 19 & 19 \\
\hline & & & 560 & 14 & 14 \\
\hline
\end{tabular}

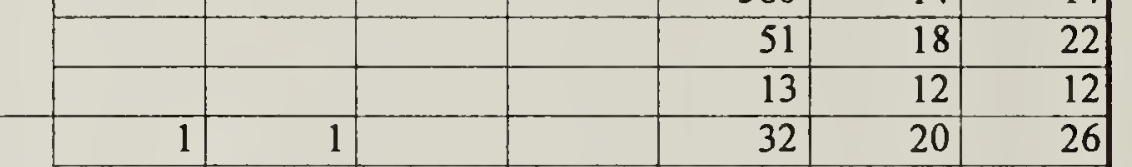

\begin{tabular}{|r|r|r|r|r|r|r|}
\hline 1 & 1 & & & 32 & 20 & 26 \\
\hline 2 & & 1 & 2 & 20 & 14 & 15 \\
\hline 1 & 43 & & & 1072 & 34 & 36 \\
\hline & & & & 162 & 16 & 17 \\
\hline & & 3 & & 121 & 36 & 40 \\
\hline
\end{tabular}


Table 4. SPECIES SEEN ON SEVEN OR FEWER COUNTS

\begin{tabular}{|c|c|}
\hline SPECIES & LOCATION and NUMBER \\
\hline \multirow{5}{*}{$\begin{array}{l}\text { PIED-BILLED GREBE } \\
\text { WESTERN GREBE } \\
\text { AMERICAN WHITE PELICAN } \\
\text { TUNDRA SWAN } \\
\text { WOOD DUCK }\end{array}$} & Fort Qu'appelle (1) \\
\hline & Coronach (1) \\
\hline & Fort Qu'appelle (1) \\
\hline & Qu'appelle Valley Dam (24), Round Lake (2) \\
\hline & Round Lake (2) \\
\hline \multirow{5}{*}{$\begin{array}{l}\text { GREEN-WINGED TEAL } \\
\text { AMERICAN BLACK DUCK } \\
\text { NORTHERN PINTAIL } \\
\text { AMERICAN WIGEON } \\
\text { REDHEAD }\end{array}$} & Fort Walsh (1), Gardiner Dam (1), Yorkton (1) \\
\hline & Coronach (2) \\
\hline & Fort Qu'appelle (1), Fort Walsh (1) \\
\hline & Coronach (1), Fort Qu'appelle (1), Yorkton (1) \\
\hline & $\begin{array}{l}\text { Coronach (2), Estevan (1), Fife Lake (1), Fort Qu'appelle (1), } \\
\text { Gardiner Dam (2), Qu'appelle Valley Dam (1) }\end{array}$ \\
\hline \multirow{5}{*}{$\begin{array}{l}\text { LESSER SCAUP } \\
\text { BUFFLEHEAD } \\
\text { HOODED MERGANSER } \\
\text { RED-BREASTED MERGANSER } \\
\text { COMMON MERGANSER }\end{array}$} & Estevan (5), Gardiner Dam (2) \\
\hline & Coronach (17), Estevan (1), Fort Qu'appelle (2) \\
\hline & Fort Qu'appelle (+), Squaw Rapids (1) \\
\hline & Yorkton (1) \\
\hline & $\begin{array}{l}\text { Estevan (1), Gardiner Dam (157), Grayson(1), Saskatoon (2), Squaw } \\
\text { Rapids (5) }\end{array}$ \\
\hline \multirow{5}{*}{$\begin{array}{l}\text { NORTHERN HARRIER } \\
\text { SHARP-SHINNED HAWK } \\
\text { COOPER'S HAWK } \\
\text { ROUGH-LEGGED HAWK }\end{array}$} & Indian Head (1) \\
\hline & Cypress Hills P.p. $(+)$ \\
\hline & Craven (1), White Bear (+) \\
\hline & $\begin{array}{l}\text { Crooked Lake (1), Estuary (1), Fort Walsh (1), Govenlock (1), Last } \\
\text { Mountain Lake N.w.a. (1) }\end{array}$ \\
\hline & Indian Head (1), Saskatoon (1) \\
\hline \multirow{2}{*}{$\begin{array}{l}\text { GYRFALCON } \\
\text { PRAIRIE FALCON }\end{array}$} & Cold Lake - Pierceland (1), Gardiner Dam (1) \\
\hline & $\begin{array}{l}\text { Govenlock (1), Grasslands N.p. (1), Harris (1), Leader South (1), } \\
\text { Rockglen (1), White Bear (+) }\end{array}$ \\
\hline \multirow[t]{2}{*}{ SPRUCE GROUSE } & $\begin{array}{l}\text { Beauval (1), Love - Torch River(5), Nipawin (+), Squaw Rapids (2), } \\
\text { Turtle Lake (2) }\end{array}$ \\
\hline & Love - Torch River (2) \\
\hline \multirow{5}{*}{$\begin{array}{l}\text { SAGE GROUSE } \\
\text { WILD TURKEY } \\
\text { AMERICAN COOT } \\
\text { KILLDEER } \\
\text { COMMON SNIPE } \\
\end{array}$} & Govenlock (21) \\
\hline & Estuary (6), Fort Walsh (8), Macdowall (2), Whitewood (+) \\
\hline & Coronach (34), Estevan (27), Fife Lake (7) \\
\hline & Saskatchewan Landing P.p. (2) \\
\hline & Fort Walsh (1) \\
\hline \multirow{5}{*}{$\begin{array}{l}\text { RING-BILLED GULL } \\
\text { HERRING GULL } \\
\text { MOURNING DOVE } \\
\text { BARRED OWL } \\
\text { GREAT GRAY OWL }\end{array}$} & Gardiner Dam (2) \\
\hline & Gardiner Dam (4) \\
\hline & Armit (1), Fort Walsh (+), Leader North (+), Preeceville (1) \\
\hline & Armit (1) \\
\hline & $\begin{array}{l}\text { Armit (1), Beauval (1), Horseshoe Bend (1), Mayview (2), Prince } \\
\text { Albert (+), Turtle Lake (2) }\end{array}$ \\
\hline \multirow{5}{*}{$\begin{array}{l}\text { LONG-EARED OWL } \\
\text { BOREAL OWL } \\
\text { BELTED KINGFISHER } \\
\text { THREE-TOED WOODPECKER }\end{array}$} & Saskatchewan Landing P.p. (1) \\
\hline & Snowden $(+)$ \\
\hline & Crooked Lake (1) \\
\hline & $\begin{array}{l}\text { Armit (2), Candle Lake (2), Duck Lake (2), Love - Torch River (+), } \\
\text { Prince Albert N.p. (2), Squaw Rapids (3) }\end{array}$ \\
\hline & Leader North $(+)$ \\
\hline \multirow{5}{*}{$\begin{array}{l}\text { AMERICAN CROW } \\
\text { MOUNTAIN CHICKADEE } \\
\text { BROWN CREEPER } \\
\text { EASTERN BLUEBIRD } \\
\text { MOUNTAIN BLUEBIRD }\end{array}$} & $\begin{array}{l}\text { Moose Mountain (1), Pike Lake (1), Regina (5), Saskatoon (13), } \\
\text { Weldon-2 (1) }\end{array}$ \\
\hline & Cypress Hills P.p. (1) \\
\hline & Biggar (1), Indian Head (1), Regina (4), Saskatoon (2), Weyburn (+) \\
\hline & Craven $(+)$ \\
\hline & Craven (+) \\
\hline \multirow{4}{*}{$\begin{array}{l}\text { TOWNSEND'S SOLITAIRE } \\
\text { VARIED THRUSH } \\
\text { CEDAR WAXWING }\end{array}$} & Fort Qu'appelle (+), Fort Walsh (1), Luseland (1), Regina (+) \\
\hline & Archerwill (+), Saskatoon (+), Waldheim (1) \\
\hline & $\begin{array}{l}\text { Craven (2), Kinistino (4)pike Lake (6), Regina (15), Saskatoon (35), } \\
\text { Yorkton (12) }\end{array}$ \\
\hline & Fort Qu'appelle (1) \\
\hline
\end{tabular}


Table 4. SPECIES SEEN ON SEVEN OR FEWER COUNTS

\begin{tabular}{|c|c|}
\hline SPECIES & LOCATION and NUMBER \\
\hline AMERICAN TREE SP & $\begin{array}{l}\text { Eastend (1), Fort Walsh (10), Leader South (4), Pike Lake (1), Skull } \\
\text { Creek (21) }\end{array}$ \\
\hline \multirow{2}{*}{$\begin{array}{l}\text { VESPER SPARROW } \\
\text { FOX SPARROW }\end{array}$} & Govenlock (1) \\
\hline & Moose Jaw (1) \\
\hline & Indian Head (1), Luseland (1) \\
\hline \multirow{2}{*}{$\begin{array}{l}\text { WHITE-CROWNED SPARROW } \\
\text { HARRIS' SPARROW }\end{array}$} & Fort Qu'appelle (t) \\
\hline & $\begin{array}{l}\text { Fort Walsh (1), Kamsack (1), Kenaston (1), Leader South (1), Regina } \\
\text { (1), Skull Creek (1), White Bear (1) }\end{array}$ \\
\hline \multirow{2}{*}{$\begin{array}{l}\text { DARK-EYED JUNCO (ORE) } \\
\text { LAPLAND LONGSPUR }\end{array}$} & Regina (3) \\
\hline & $\begin{array}{l}\text { Bromhead (1298), Fife Lake (120), Rockglen (5), Weyburn (127), } \\
\text { Whitewood (5) }\end{array}$ \\
\hline \multirow{3}{*}{$\begin{array}{l}\text { RED-WINGED BLACKBIRD } \\
\text { WESTERN MEADOWLARK } \\
\text { RUSTY BLACKBIRD }\end{array}$} & Fort $\mathrm{Wa}$ \\
\hline & Kutawagan Lake (1), Rockglen (+) \\
\hline & $\begin{array}{l}\text { Biggar (1), Bradview (3), Clark's Crossing (1), Regina (+), Skull } \\
\text { Creek (1), Whitewood (7) }\end{array}$ \\
\hline BREWER'S BLACKBIRD & Archerwill (1), Endeavour (1), Pike Lake (2) \\
\hline \multirow{3}{*}{$\begin{array}{l}\text { COMMON GRACKLE } \\
\text { GRAY-CROWNED ROSY FINCH } \\
\text { PURPLE FINCH }\end{array}$} & $\begin{array}{l}\text { Bangor (+), Kamsack (1), Regina (15), Saskatoon (3), Swift Current } \\
\text { (1), Weyburn (1) }\end{array}$ \\
\hline & Fort Walsh $(19)$, Rockglen $(+)$ \\
\hline & $\begin{array}{l}\text { Fort Qu'appelle (+), Kenosee Lake (10), Regina (1), Swift Current } \\
\text { (36), Tisdale (7), Weyburn (2) }\end{array}$ \\
\hline \multirow{2}{*}{$\begin{array}{l}\text { WHITE-WINGED CROSSBILL } \\
\text { AMERICAN GOLDFINCH }\end{array}$} & Cold Lake - Pierceland (18), Raymore (+), Saskatoon (25) \\
\hline & $\begin{array}{l}\text { Craven (2), Kenosee Lake (9), Moose Mountain (3), Regina (1), } \\
\text { Saskatoon (1), Whitewood (3) }\end{array}$ \\
\hline
\end{tabular}

Table 5. BIRDS NOT IDENTIFIED TO SPECIES

\begin{tabular}{|l|l|}
\hline SPECIES & LOCATION and NUMBER \\
\hline DARK GOOSE species & Gardiner Dam (74) \\
\cline { 2 - 2 } BUTEO species & Cypress Hills P.p. (1) \\
\cline { 2 - 2 } EAGLE species & Gardiner Dam (2), Saskatoon (1) \\
\cline { 2 - 2 } HAWK species & Estuary (1) \\
\hline WOODPECKER species & Fort Walsh (1) \\
\cline { 2 - 2 } BLACKBIRD species & Saskatchewan Landing P.p. ( + ) \\
\cline { 2 - 2 } REDPOLL species & Fort Walsh (17). Saskatchewan Landing P.p. (250) \\
\hline
\end{tabular}


Table 6: NEW AND TYING 1998 HIGH COUNTS (new highs in bold italic)

\begin{tabular}{|c|c|c|c|c|}
\hline LOCATION & $\begin{array}{c}1998 \\
\text { COUNT }\end{array}$ & SPECIES & $\begin{array}{c}\text { PREVIOUS } \\
\text { HIGH }\end{array}$ & LOCATION (YEAR) \\
\hline Coronach & 1 & WESTERN GREBE & 1 & N. to Gardiner Dam \\
\hline Qu'appelle Dam & 24 & TUNDRA SWAN & 8 & Regina (1965) \\
\hline Round Lake & 2 & WOOD DUCK & 1 & Gardiner Dam (1988) \\
\hline Coronach & 2 & AMERICAN BLACK DUCK & 2 & Regina $(1992,93,94)$ \\
\hline Coronach & 17 & BUFFLEHEAD & 7 & Coronach (1997) \\
\hline Craven & 1 & COOPER'S HAWK & 1 & N. to Kamsack \\
\hline $\begin{array}{l}\text { Indian Head, } \\
\text { Saskatoon }\end{array}$ & 1 & AMERICAN KESTREL & 1 & N. to Dalmeny \\
\hline Snowden & 24 & RUFFED GROUSE & 20 & Armit \&Kamsack (1997) \\
\hline Sk. Landing P.P. & 2 & KILLDEER & 1 & Govenlock $(1982,90)$ \\
\hline Pike Lake & 39 & HAIRY WOODPECKER & 36 & Saskatoon (1991) \\
\hline Saskatoon & 111 & BLUE JAY & 111 & Saskatoon (1995) \\
\hline Saskatoon & 976 & $B L A C K-B I L L E D$ MAGPIE & 670 & Saskatoon (1995) \\
\hline Saskatoon & 640 & $B L A C K-C A P P E D$ CHICKADEE & 461 & Saskatoon (1995) \\
\hline Craven (count period) & 0 & EASTERN BLUEBIRD & New & \\
\hline Waldheim & 1 & VARIED THRUSH & 1 & North to Saskatoon \\
\hline Moose Jaw & 1 & FOX SPARROW & 1 & North to Saskatoon \\
\hline Regina & 15 & COMMON GRACKLE & 11 & Regina (1991) \\
\hline Swift Current & 36 & PURPLE FINCH & 19 & Swift Current (1997) \\
\hline Regina & 190 & HOUSE FINCH & 78 & Regina (1996) \\
\hline Kenosee Lake & 9 & AMERICAN GOLDFINCH & 2 & Craven (1995) \\
\hline
\end{tabular}

Table 7: POPULATION STATUS OF SELECTED SPECIES (based on birds per hours effort)

\begin{tabular}{|c|c|c|c|c|c|c|c|}
\hline PECIES & 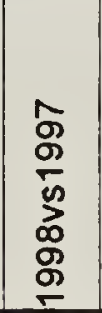 & 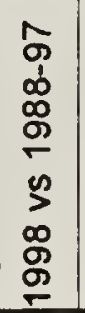 & 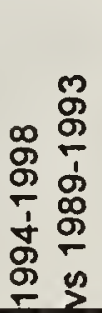 & SPECIES & 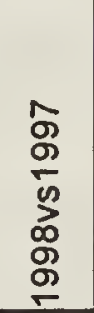 & $\begin{array}{l}1 \\
0 \\
0 \\
0 \\
0 \\
o \\
0 \\
0 \\
0 \\
0 \\
0 \\
0 \\
\end{array}$ & 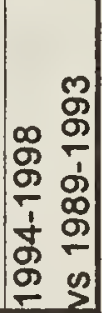 \\
\hline \multirow{5}{*}{$\begin{array}{l}\text { CANADA GOOSE } \\
\text { MALLARD } \\
\text { COMMON GOLDENEYE } \\
\text { BALD EAGLE } \\
\text { NORTHERN GOSHAWK }\end{array}$} & -94 & -58 & 896 & \multirow{5}{*}{$\begin{array}{l}\text { COMMON RAVEN } \\
\text { BLACK-CAPPED CHICKADEE } \\
\text { BOREAL CHICKADEE } \\
\text { RED-BREASTED NUTHATCH } \\
\text { WHITE-BREASTED NUTHATCH }\end{array}$} & 10 & 42 & \\
\hline & -29 & 40 & 119 & & 18 & 29 & \\
\hline & -33 & -21 & 2 & & -15 & 20 & 38 \\
\hline & -65 & -26 & 98 & & -69 & -30 & 153 \\
\hline & 53 & -31 & -46 & & 5 & 14 & 22 \\
\hline \multirow{5}{*}{$\begin{array}{l}\text { GOLDEN EAGLE } \\
\text { MERLIN } \\
\text { PRAIRIE FALCON } \\
\text { GRAY PARTRIDGE } \\
\text { RING-NECKED PHEASANT } \\
\end{array}$} & -23 & -35 & -20 & \multirow{5}{*}{$\begin{array}{l}\text { BROWN CREEPER } \\
\text { GOLDEN-CROWNED KINGLET } \\
\text { AMERICAN ROBIN } \\
\text { BOHEMIAN WAXWING } \\
\text { CEDAR WAXWING }\end{array}$} & -6 & 28 & \\
\hline & -22 & 15 & 46 & & -66 & -21 & \\
\hline & 32 & -27 & -55 & & .38 & -68 & \\
\hline & 115 & -32 & -7 & & 46 & 119 & 28 \\
\hline & 201 & -0 & -36 & & -84 & -86 & 40 \\
\hline \multirow{5}{*}{$\begin{array}{l}\text { SPRUCE GROUSE } \\
\text { RUFFED GROUSE } \\
\text { SHARP-TAILED GROUSE } \\
\text { ROCK DOVE } \\
\text { GREAT HORNED OWL }\end{array}$} & 958 & 7 & 51 & \multirow{5}{*}{$\begin{array}{l}\text { NORTHERN SHRIKE } \\
\text { EUROPEAN STARLING } \\
\text { AMERICAN TREE SPARROW } \\
\text { DARK-EYED JUNCO } \\
\text { LAPLAND LONGSPUR }\end{array}$} & 21 & -38 & \\
\hline & -19 & 12 & 32 & & 5 & -15 & 118 \\
\hline & 1 & -18 & -10 & & 118 & 13 & -43 \\
\hline & -20 & -15 & 3 & & 0 & -2 & 71 \\
\hline & -30 & -26 & -7 & & 5777 & -29 & -41 \\
\hline \multirow{5}{*}{$\begin{array}{l}\text { SNOWY OWL } \\
\text { SHORT-EARED OWL } \\
\text { DOWNY WOODPECKER } \\
\text { HAIRY WOODPECKER } \\
\text { THREE-TOED WOODPECKER }\end{array}$} & -58 & -41 & 6 & \multirow{5}{*}{$\begin{array}{l}\text { SNOW BUNTING } \\
\text { RUSTY BLACKBIRD } \\
\text { PINE GROSBEAK } \\
\text { HOUSE FINCH } \\
\text { RED CROSSBILL }\end{array}$} & 447 & 18 & -20 \\
\hline & 394 & \begin{tabular}{|l|}
-11 \\
\end{tabular} & -40 & & 129 & -63 & -25 \\
\hline & 31 & 19 & 4 & & -23 & -27 & \\
\hline & 28 & 17 & 11 & & 198 & 519 & 2845 \\
\hline & -23 & -7 & 57 & & -4 & 143 & 262 \\
\hline \multirow{6}{*}{$\begin{array}{l}\text { NORTHERN FLICKER } \\
\text { PILEATED WOODPECKER } \\
\text { HORNED LARK } \\
\text { GRAY JAY } \\
\text { BLUE JAY } \\
\text { BLACK-BILLED MAGPIE }\end{array}$} & -44 & 9 & 119 & \multirow{6}{*}{$\begin{array}{l}\text { WHITE-WINGED CROS } \\
\text { COMMON REDPOLL } \\
\text { HOARY REDPOLL } \\
\text { PINE SISKIN } \\
\text { EVENING GROSBEAK } \\
\text { HOUSE SPARROW }\end{array}$} & -63 & -87 & \\
\hline & 26 & -13 & -22 & & 37 & -11 & -23 \\
\hline & 171 & 41 & -52 & & 104 & -36 & -7 \\
\hline & 7 & -2 & 2 & & -10 & 188 & 138 \\
\hline & 7 & 32 & 28 & & -64 & -59 & -19 \\
\hline & 11 & 33 & 26 & & & 11 & \\
\hline
\end{tabular}

